\title{
Light-responsive Serinol-based Polycarbonate and Polyester as Degradable Scaffolds
}

Jingjiang Sun ${ }^{a, b}$, Dimitri Jung, ${ }^{b}$ Timo Schoppa ${ }^{c}$, Juliane Anderskic, Marie-Theres

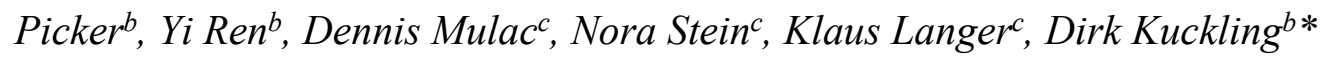

aKey Laboratory of Rubber-plastics, Ministry of Education/Shandong

Provincial Key Laboratory of Rubber-plastics, School of Polymer Science and Engineering, Qingdao University of Science and Technology, Zhengzhou Rd. 53, CN266042 Qingdao, China

bepartment of Chemistry, Paderborn University, Warburger Str. 100, D-33098

Paderborn, Germany

Email: dirk.kuckling@uni-paderborn.de

'Institute of Pharmaceutical Technology and Biopharmacy, University of Münster, Corrensstr. 48, D-48149 Münster, Germany

\section{Supporting Information}




\section{EXPERIMENTAL SECTION}

Materials. 1,4-Butanediol ( $>99 \%$, Acros Organics) was vacuum distilled using a short path distillation apparatus and dried over $4 \AA$ molecular sieves. Dichloromethane (DCM) (98\%, Stockmeier Chemie) was dried over $\mathrm{CaCl}_{2}$ then distilled over calcium hydride. Tetrahydrofuran (THF) (98\%, Stockmeier Chemie) was dried over KOH then distilled over calcium hydride. Acetonitrile $(\mathrm{MeCN})(>99.9 \%$, Carl Roth), adipoyl chloride (98\%, Alfa Aesar), diisopropylethylamine (DIPEA) (99\%, Alfa Aesar), 4,5dimethoxy-2-nitrobenzyl alcohol (98\%, Alfa Aesar), N,N-dimethylformamide (DMF) (99.8\%, Acros Organics), di-tert-butyl dicarbonate (97\%, Acros Organics), ethyl chloroformate ( $>98 \%$, Fluka), hydrogen chloride (HCl) 4 M in 1,4-dioxane (99\%, Alfa Aesar), 4-nitrophenyl chloroformate (97\%, abcr), pyridine (99.5\%, Acros Organics), serinol (97\%, abcr), triethylamine (TEA) (99\%, Acros Organics), triphosgene (98\%, abcr) were used as received. For nanoparticle preparation poly(vinyl alcohol) (PVA; average molecular weight $30-70 \mathrm{kDa}, 87-90 \%$ hydrolyzed) was purchased from Sigma-Aldrich (Steinheim, Germany), and dichloromethane and ethyl acetate were achieved from VWR (Darmstadt, Germany).

Measurements. ${ }^{1} \mathrm{H}$ and ${ }^{13} \mathrm{C}$ nuclear magnetic resonance (NMR) spectra were recorded using Bruker AV 500 spectrometer at $500 \mathrm{MHz}$ and $125 \mathrm{MHz}$, respectively. Chloroform- $d\left(\mathrm{CDCl}_{3}-d, 99.8 \mathrm{D} \%\right)$, deuterium oxide- $d_{2}\left(\mathrm{D}_{2} \mathrm{O}-d_{2} \quad 99.5 \mathrm{D} \%\right)$ or dimethylsulfoxide- $d_{6}$ (DMSO- $d_{6}, 99.8 \mathrm{D} \%$ ) were used as solvents for NMR measurements. Electrospray ionization time-of-flight (ESI-ToF)-mass spectra were measured on a SYNAPT G2 HDMS ${ }^{\mathrm{TM}}$ from Waters. Data were obtained with Mass Lynx 4.1. Attenuated total reflection-Fourier transform infrared (ATR-FTIR) spectra were recorded on Vertex 70 spectrometer (Bruker Optik, Ettlingen, Germany) with a RT_DLaTGS Detector. All samples were measured in the wavenumber region of $4,000 \mathrm{~cm}^{-1}$ to $400 \mathrm{~cm}^{-1}$. Melting temperature $\left(\mathrm{T}_{\mathrm{m}}\right)$ of monomers was measured by employing Büchi Melting Point B-545. The molar masses and dispersities $\left(\bigoplus_{M}\right)$ were analyzed employing a size exclusion chromatography (SEC) system (advanced 
polymer chromatography from Waters) equipped with two consecutive columns (Acquity APC XT columns filled with polyethoxysilane with a defined porosity of $125 \AA$ and $45 \AA$, respectively) and an Acquity APC RI-detector. The system was operated at a flow rate of $0.7 \mathrm{~mL} / \mathrm{min}$ with $\mathrm{THF} / \mathrm{DMF}(\mathrm{v} / \mathrm{v}=80 / 20)$ as solvents. Poly(methyl methacrylate) (PMMA) standards were used for calibration. Differential scanning calorimetry (DSC) was performed with Netzsch DSC 204 F1 Phönix at a heating rate of $10 \mathrm{~K} / \mathrm{min}$ under a nitrogen atmosphere. The glass transition temperature $\left(\mathrm{T}_{\mathrm{g}}\right)$ and melting temperature $\left(\mathrm{T}_{\mathrm{m}}\right)$ values of polymers were recorded during the second run. Light degradation experiments were performed using an OmniCure S1500 Curing System from Lumen Dynamics with a power of $0.196 \mathrm{~W} / \mathrm{cm}^{2}(320-480 \mathrm{~nm})$. UV/Vis spectra were recorded on Specord 50 PLUS UV/Vis spectrophotometer from Analytik Jena using Aspect UV 1.1 software.

\section{Synthesis of small molecules}<smiles>COc1cc(CO)c([N+](=O)[O-])cc1COC(=O)Oc1ccc([N+](=O)[O-])cc1</smiles>

1

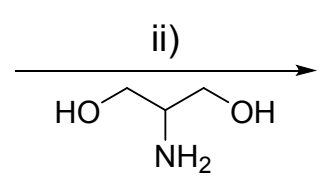

serinol<smiles>COc1cc(COC(=O)NC(CO)CO)c([N+](=O)[O-])cc1OC</smiles>

3

Scheme S1. Synthetic route of the oNB containing diol 3. Reagents and conditions: i) 4-nitrophenyl chloroformate, DIPEA, DCM, rt, overnight, 91\%; ii) serinol, TEA, $\mathrm{MeCN}$, rt, overnight, $77 \%$.

Synthesis of 4,5-dimethoxy-2-nitrobenzyl(4-nitrophenyl)carbonate (2) ${ }^{1}$

In a round-flask 4,5-dimethoxy-2-nitrobenzyl alcohol (1) $(8.80 \mathrm{~g}, 41.3 \mathrm{mmol})$ and 4- 
nitrophenyl chloroformate $(15.55 \mathrm{~g}, 77.2 \mathrm{mmol})$ were suspended in DCM $(90 \mathrm{~mL})$. After adding DIPEA $(15 \mathrm{~mL}, 86.1 \mathrm{mmol})$ the reaction mixture was stirred at room temperature for $1.5 \mathrm{~h}$, diluted with DCM $(90 \mathrm{~mL})$ and stirred overnight. The solvent was removed by rotary evaporator. The residue was suspended in EtOH $(290 \mathrm{~mL})$ and refluxed for $45 \mathrm{~min}$. The precipitate was filtered off, washed with hot EtOH and dried in vacuo to afford $14.16 \mathrm{~g}$ of a yellowish solid (yield $=91 \%$ ).

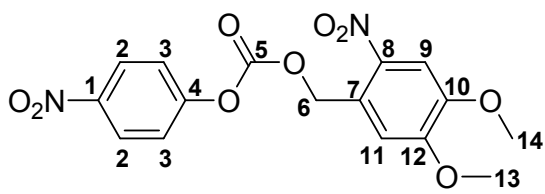

Scheme S2. Structure of compound 2 for the structural assignment from the NMR result.

${ }^{1} \mathrm{H}-\mathrm{NMR}\left(500 \mathrm{MHz}, \mathrm{DMSO}-d_{6}\right): \delta(\mathrm{ppm})=3.89\left(\mathrm{~s}, 3 \mathrm{H},{ }^{13} \mathrm{CH}_{3}\right), 3.92\left(\mathrm{~s}, 3 \mathrm{H},{ }^{14} \mathrm{CH}_{3}\right)$, $5.61\left(\mathrm{~s}, 2 \mathrm{H},{ }^{6} \mathrm{CH}_{2}\right), 7.26\left(\mathrm{~s}, 1 \mathrm{H},{ }^{11} \mathrm{CH}\right), 7.59\left(\mathrm{~d},{ }^{3} \mathrm{~J}_{\mathrm{HH}}=9.1 \mathrm{~Hz}, 2 \mathrm{H},{ }^{3} \mathrm{CH}\right), 7.73(\mathrm{~s}, 1 \mathrm{H}$, $\left.{ }^{9} \mathrm{CH}\right), 8.33\left(\mathrm{~d},{ }^{3} \mathrm{~J}_{\mathrm{HH}}=9.1 \mathrm{~Hz}, 2 \mathrm{H},{ }^{2} \mathrm{CH}\right)$.

${ }^{13} \mathrm{C}-\mathrm{NMR}\left(125 \mathrm{MHz}, \mathrm{DMSO}-d_{6}\right): \delta(\mathrm{ppm})=56.14\left(1 \mathrm{C},{ }^{13} \mathrm{CH}_{3}\right), 56.32\left(1 \mathrm{C},{ }^{14} \mathrm{CH}_{3}\right)$, $67.22\left(1 \mathrm{C},{ }^{6} \mathrm{CH}_{2}\right), 108.30\left(1 \mathrm{C},{ }^{9} \mathrm{CH}\right), 112.00\left(1 \mathrm{C},{ }^{11} \mathrm{CH}\right), 122.57\left(2 \mathrm{C},{ }^{3} \mathrm{CH}\right), 124.37$ $\left(1 \mathrm{C},{ }^{7} \mathrm{C}_{\mathrm{q}}\right), 125.45\left(2 \mathrm{C},{ }^{2} \mathrm{CH}\right), 139.94\left(1 \mathrm{C},{ }^{8} \mathrm{C}_{\mathrm{q}}\right), 145.25\left(1 \mathrm{C},{ }^{1} \mathrm{C}_{\mathrm{q}}\right), 148.40\left(1 \mathrm{C},{ }^{12} \mathrm{C}_{\mathrm{q}}\right)$, $151.62\left(1 \mathrm{C},{ }^{5} \mathrm{C}_{\mathrm{q}}\right), 153.17\left(1 \mathrm{C},{ }^{10} \mathrm{C}_{\mathrm{q}}\right), 155.17\left(1 \mathrm{C},{ }^{4} \mathrm{C}_{\mathrm{q}}\right) .($ Scheme S2)

Synthesis of 4,5-dimethoxy-2-nitrobenzyl (1,3-dihydroxypropan-2-yl)carbamate (3)

In a round bottom flask serinol $(2.94 \mathrm{~g}, 27.8 \mathrm{mmol}), 2(7.00 \mathrm{~g}, 18.5 \mathrm{mmol})$ and TEA $(9.66 \mathrm{~mL}, 69.4 \mathrm{mmol})$ were suspended in dry $\mathrm{MeCN}(140 \mathrm{~mL})$. The reaction mixture was stirred at room temperature overnight. The precipitate was collected by filtration and washed with DCM $(150 \mathrm{~mL})$. In the case of low yield, the filtrate was concentrated to ca. $50 \mathrm{~mL}$ under reduced pressure. The precipitate was filtered off and recrystallized from acetone. The product was collected and dried in vacuo to achieve $4.93 \mathrm{~g}$ of an offwhite solid (yield $=77 \%$ ). 


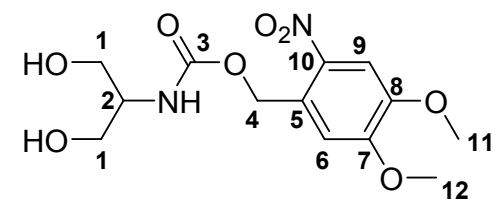

Scheme S3. Structure of compound 3 for the structural assignment from the NMR result.

${ }^{1} \mathrm{H}-\mathrm{NMR}\left(500 \mathrm{MHz}, \mathrm{DMSO}-d_{6}\right): \delta(\mathrm{ppm})=3.35-3.60\left(\mathrm{~m}, 4 \mathrm{H},{ }^{1} \mathrm{CH}_{2} ; 1 \mathrm{H},{ }^{2} \mathrm{CH}\right), 3.87$ $\left(\mathrm{s}, 3 \mathrm{H},{ }^{12} \mathrm{CH}_{3}\right), 3.91\left(\mathrm{~s}, 3 \mathrm{H},{ }^{11} \mathrm{CH}_{3}\right), 4.59\left(\mathrm{t},{ }^{3} \mathrm{~J}_{\mathrm{HH}}=5.4 \mathrm{~Hz}, 2 \mathrm{H}, \mathrm{OH}\right), 5.33(\mathrm{~s}, 2 \mathrm{H}$, $\left.{ }^{4} \mathrm{CH}_{2}\right), 7.14\left(\mathrm{~d},{ }^{3} \mathrm{~J}_{\mathrm{HH}}=8.0 \mathrm{~Hz}, 1 \mathrm{H}, \mathrm{NH}\right), 7.21\left(\mathrm{~s}, 1 \mathrm{H},{ }^{6} \mathrm{CH}\right), 7.70\left(\mathrm{~s}, 1 \mathrm{H},{ }^{9} \mathrm{CH}\right)$.

${ }^{13} \mathrm{C}-\mathrm{NMR}\left(125 \mathrm{MHz}, \mathrm{DMSO}-d_{6}\right): \delta(\mathrm{ppm})=55.13\left(1 \mathrm{C},{ }^{2} \mathrm{CH}\right), 56.21\left(1 \mathrm{C},{ }^{12} \mathrm{CH}_{3}\right)$, $56.39\left(1 \mathrm{C},{ }^{11} \mathrm{CH}_{3}\right), 60.60\left(2 \mathrm{C},{ }^{1} \mathrm{CH}_{2}\right), 62.32\left(1 \mathrm{C},{ }^{4} \mathrm{CH}_{2}\right), 108.25\left(1 \mathrm{C},{ }^{9} \mathrm{CH}\right), 110.40(1$ $\left.\mathrm{C},{ }^{6} \mathrm{CH}\right), 128.40\left(1 \mathrm{C},{ }^{5} \mathrm{C}_{\mathrm{q}}\right), 139.20\left(1 \mathrm{C},{ }^{10} \mathrm{C}_{\mathrm{q}}\right), 147.74\left(1 \mathrm{C},{ }^{7} \mathrm{C}_{\mathrm{q}}\right), 153.61\left(1 \mathrm{C},{ }^{8} \mathrm{C}_{\mathrm{q}}\right)$, $155.63\left(1 \mathrm{C},{ }^{3} \mathrm{C}_{\mathrm{q}}\right)$. (Scheme S3)

ESI-ToF-MS (m/z): $[\mathrm{M}+\mathrm{Na}]^{+}$calculated for $\mathrm{C}_{13} \mathrm{H}_{18} \mathrm{~N}_{2} \mathrm{O}_{8} \mathrm{Na}^{+}$, 353.0961; found, 353.0959 .

IR $\left(\mathrm{ATR}, \mathrm{cm}^{-1}\right): 3,308(v(\mathrm{O}-\mathrm{H})$ and $v(\mathrm{~N}-\mathrm{H})), 1,689(v(\mathrm{C}=\mathrm{O}$, carbamate $)), 1,580(v$ $(\mathrm{C}=\mathrm{C}$, aromatic $)), 1,520\left(v_{a s}\left(\mathrm{NO}_{2}\right)\right), 1,332\left(v_{s}\left(\mathrm{NO}_{2}\right)\right), 1,276(v(\mathrm{C}-\mathrm{O}$, carbamate $))$ and $1,220(v(\mathrm{C}-\mathrm{O}$, ether $))$.

Melting point $\left(\mathrm{T}_{\mathrm{m}}\right): 147.4^{\circ} \mathrm{C}$

Synthesis of 4,5-dimethoxy-2-nitrobenzyl (2-oxo-1,3-dioxan-5-yl)carbamate (4)

In a round bottom flask $3(1.83 \mathrm{~g}, 5.6 \mathrm{mmol})$ was suspended in THF $(16 \mathrm{~mL})$. Ethyl chloroformate $(1.59 \mathrm{~mL}, 16.6 \mathrm{mmol})$ and TEA $(2.67 \mathrm{~mL}, 19.1 \mathrm{mmol})$ were added dropwise at $0{ }^{\circ} \mathrm{C}$. The reaction mixture was stirred at room temperature overnight. The precipitate was collected, washed with THF $(15 \mathrm{~mL})$, stirred in water $(50 \mathrm{~mL})$ for $30 \mathrm{~min}$, filtered off, washed with water $(50 \mathrm{~mL})$ and $\mathrm{Et}_{2} \mathrm{O}(100 \mathrm{~mL})$, recrystallized from DMF $(13 \mathrm{~mL} / \mathrm{g})$ and dried in vacuo to afford $1.27 \mathrm{~g}$ of an off-white solid (yield $=64 \%$ ). 


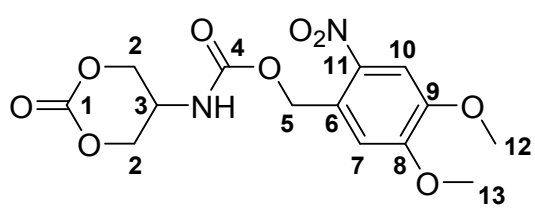

Scheme S4. Structure of compound 4 for the structural assignment from the NMR result.

${ }^{1} \mathrm{H}-\mathrm{NMR}\left(500 \mathrm{MHz}, \mathrm{DMSO}-d_{6}\right): \delta(\mathrm{ppm})=3.87\left(\mathrm{~s}, 3 \mathrm{H},{ }^{13} \mathrm{CH}_{3}\right), 3.91\left(\mathrm{~s}, 3 \mathrm{H},{ }^{12} \mathrm{CH}_{3}\right)$, $4.04\left(\mathrm{~m}, 1 \mathrm{H},{ }^{3} \mathrm{CH}\right), 4.30\left(\mathrm{~d},{ }^{3} \mathrm{~J}_{\mathrm{HH}}=10.4 \mathrm{~Hz}, 2 \mathrm{H},{ }^{2} \mathrm{CH}_{2}\right), 4.60\left(\mathrm{~d},{ }^{3} \mathrm{~J}_{\mathrm{HH}}=10.4 \mathrm{~Hz}, 2 \mathrm{H}\right.$, $\left.{ }^{2} \mathrm{CH}_{2}\right), 5.38\left(\mathrm{~s}, 2 \mathrm{H},{ }^{5} \mathrm{CH}_{2}\right), 7.21\left(\mathrm{~s}, 1 \mathrm{H},{ }^{7} \mathrm{CH}\right), 7.71\left(\mathrm{~s}, 1 \mathrm{H},{ }^{10} \mathrm{CH}\right), 8.27(\mathrm{~s}, 1 \mathrm{H}, \mathrm{NH})$.

${ }^{13} \mathrm{C}-\mathrm{NMR}\left(125 \mathrm{MHz}, \mathrm{DMSO}-d_{6}\right): \delta(\mathrm{ppm})=42.40\left(1 \mathrm{C},{ }^{3} \mathrm{CH}\right), 56.23\left(1 \mathrm{C},{ }^{13} \mathrm{CH}_{3}\right)$, $56.39\left(1 \mathrm{C},{ }^{12} \mathrm{CH}_{3}\right), 62.84\left(1 \mathrm{C},{ }^{5} \mathrm{CH}_{2}\right), 70.14\left(2 \mathrm{C},{ }^{2} \mathrm{CH}_{2}\right), 108.33\left(1 \mathrm{C},{ }^{10} \mathrm{CH}\right), 110.74$ $\left(1 \mathrm{C},{ }^{7} \mathrm{CH}\right), 127.54\left(1 \mathrm{C},{ }^{6} \mathrm{C}_{\mathrm{q}}\right), 139.42\left(1 \mathrm{C},{ }^{11} \mathrm{C}_{\mathrm{q}}\right), 147.39\left(1 \mathrm{C},{ }^{1} \mathrm{C}_{\mathrm{q}}\right), 147.93\left(1 \mathrm{C},{ }^{8} \mathrm{C}_{\mathrm{q}}\right)$, $153.52\left(1 \mathrm{C},{ }^{9} \mathrm{C}_{\mathrm{q}}\right), 155.65\left(1 \mathrm{C},{ }^{4} \mathrm{C}_{\mathrm{q}}\right) .($ Scheme $\mathrm{S4})$

ESI-ToF-MS (m/z): $[\mathrm{M}+\mathrm{Na}]^{+}$calculated for $\mathrm{C}_{14} \mathrm{H}_{16} \mathrm{~N}_{2} \mathrm{O}_{9} \mathrm{Na}^{+}$, 379.0753; found, 379.0743 .

IR $\left(\mathrm{ATR}, \mathrm{cm}^{-1}\right): 3,287(v(\mathrm{~N}-\mathrm{H})), 1,744(v(\mathrm{C}=\mathrm{O}$, carbonate $)), 1,709(v(\mathrm{C}=\mathrm{O}$, carbamate) $), 1,580(v(\mathrm{C}=\mathrm{C}$, aromatic $)), 1,524\left(v_{a s}\left(\mathrm{NO}_{2}\right)\right), 1,313\left(v_{s}\left(\mathrm{NO}_{2}\right)\right), 1,277(v$ (C-O, carbamate) $)$ and 1,218 (v (C-O, ether $))$.

Melting point $\left(\mathrm{T}_{\mathrm{m}}\right): 162.4{ }^{\circ} \mathrm{C}$

Synthesis of tert-butyl (1,3-dihydroxypropan-2-yl)carbamate (5) ${ }^{2}$

In a round bottom flask serinol $(3.0 \mathrm{~g}, 33 \mathrm{mmol})$ and TEA $(6.8 \mathrm{~mL}, 50 \mathrm{mmol})$ were dissolved in a mixture of water and THF $(30 \mathrm{~mL}, 4 / 1, \mathrm{v} / \mathrm{v})$. A solution of di-tert-butyl dicarbonate $(8.7 \mathrm{~g}, 39 \mathrm{mmol})$ in THF $(6 \mathrm{~mL})$ was dropped into the flask over $1 \mathrm{~h}$. The reaction mixture was then stirred at room temperature for $3 \mathrm{~h}$. After removing the THF under reduced pressure, the residue was extracted four times with ethyl acetate $(4 \mathrm{x}$ $200 \mathrm{~mL}$ ). The collected organic phase was dried over anhydrous $\mathrm{MgSO}_{4}$ and the solvent was removed under reduced pressure. The residue was purified by recrystallization from ethyl acetate to afford $6.30 \mathrm{~g}$ of a colorless solid (yield $=84 \%$ ). 


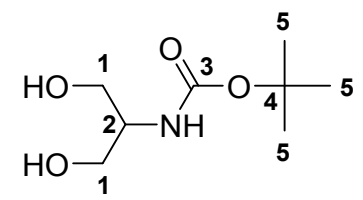

Scheme S5. Structure of compound 5 for the structural assignment from the NMR result.

${ }^{1} \mathrm{H}-\mathrm{NMR}\left(500 \mathrm{MHz}, \mathrm{DMSO}-d_{6}\right): \delta(\mathrm{ppm})=1.38\left(\mathrm{~s}, 9 \mathrm{H},{ }^{5} \mathrm{CH}_{3}\right), 3.30-3.40(\mathrm{~m}, 4 \mathrm{H}$, $\left.{ }^{1} \mathrm{CH}_{2} ; 1 \mathrm{H},{ }^{2} \mathrm{CH}\right), 4.48\left(\mathrm{t},{ }^{3} \mathrm{~J}_{\mathrm{HH}}=5.1 \mathrm{~Hz}, 2 \mathrm{H}, \mathrm{OH}\right), 6.25(\mathrm{~s}, 1 \mathrm{H}, \mathrm{NH})$.

${ }^{13} \mathrm{C}-\mathrm{NMR}\left(125 \mathrm{MHz}, \mathrm{DMSO}-d_{6}\right): \delta(\mathrm{ppm})=28.34\left(3 \mathrm{C},{ }^{5} \mathrm{CH}_{3}\right), 54.39\left(1 \mathrm{C},{ }^{2} \mathrm{CH}\right), 60.61$ $\left(2 \mathrm{C},{ }^{1} \mathrm{CH}_{2}\right), 77.62\left(1 \mathrm{C},{ }^{4} \mathrm{C}_{\mathrm{q}}\right), 155.39\left(1 \mathrm{C},{ }^{3} \mathrm{C}_{\mathrm{q}}\right)$. (Scheme S5)

ESI-ToF-MS (m/z): [M+Na] $]^{+}$calculated for $\mathrm{C}_{8} \mathrm{H}_{17} \mathrm{NO}_{4} \mathrm{Na}^{+}$, 214.1055; found, 214.1056. 

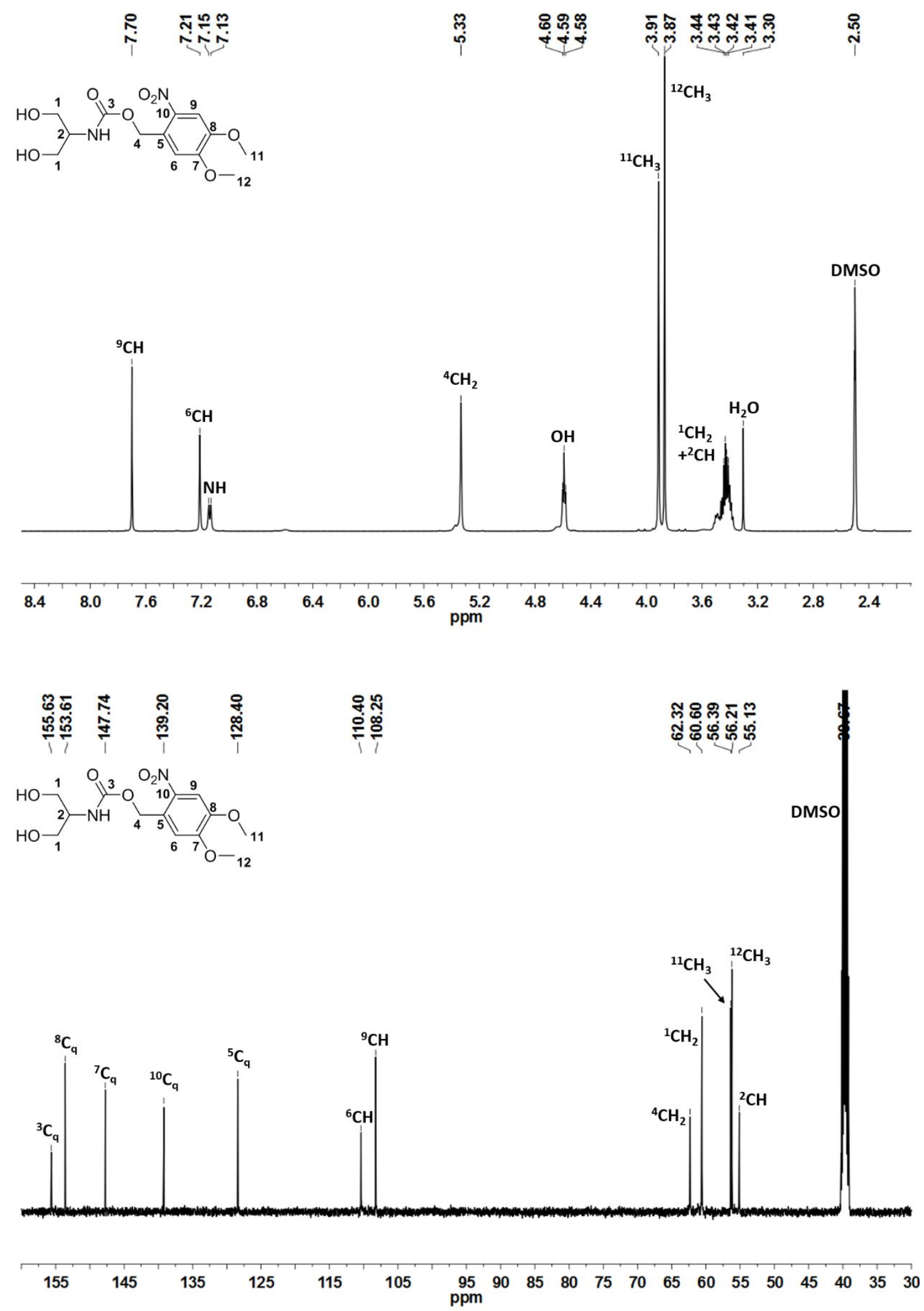

Figure S1. ${ }^{1} \mathrm{H}$ and ${ }^{13} \mathrm{C}$ NMR spectra of compound $\mathbf{3}$ 

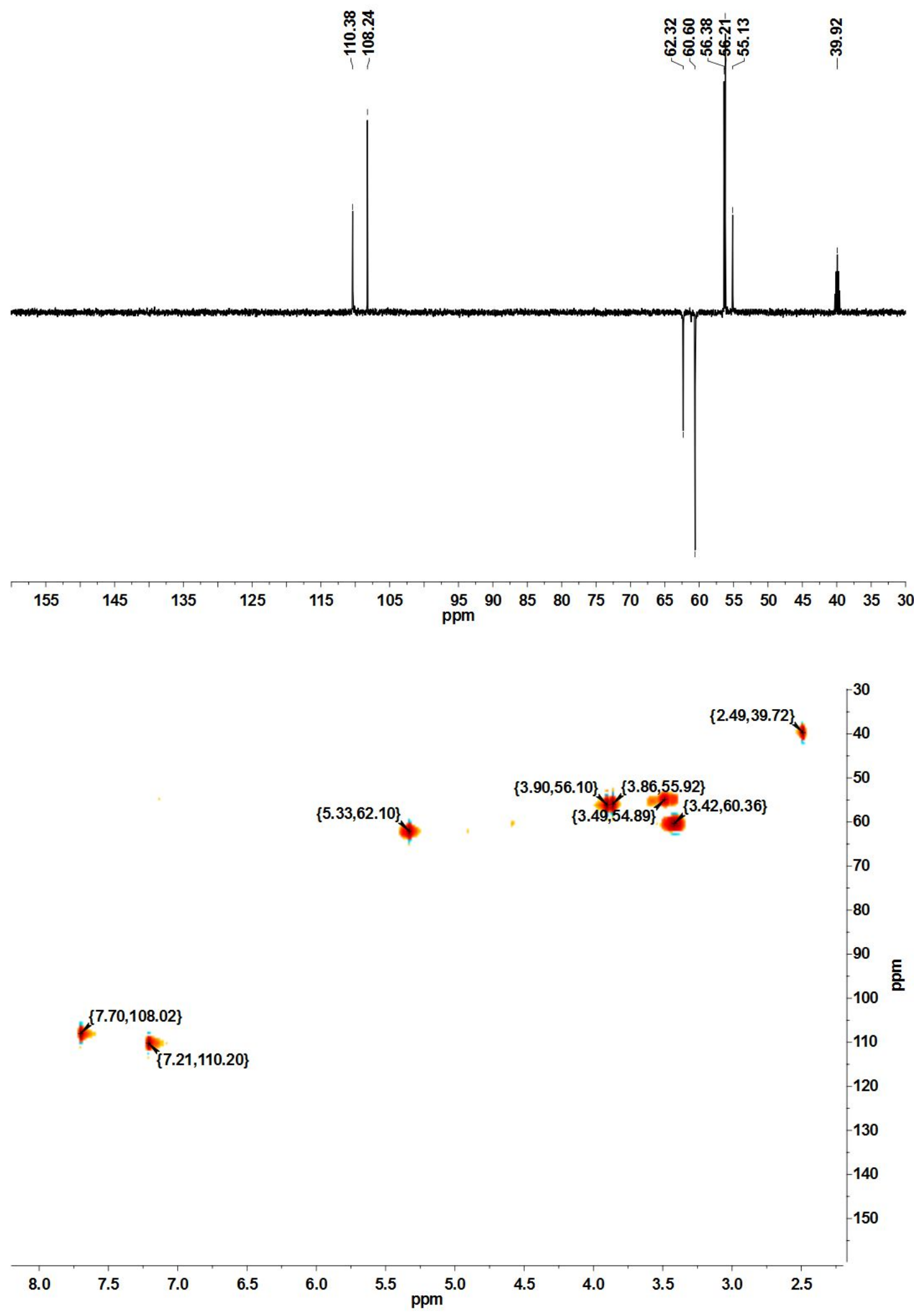

Figure S2. DEPT 135 and HSQC NMR spectra of compound 3 

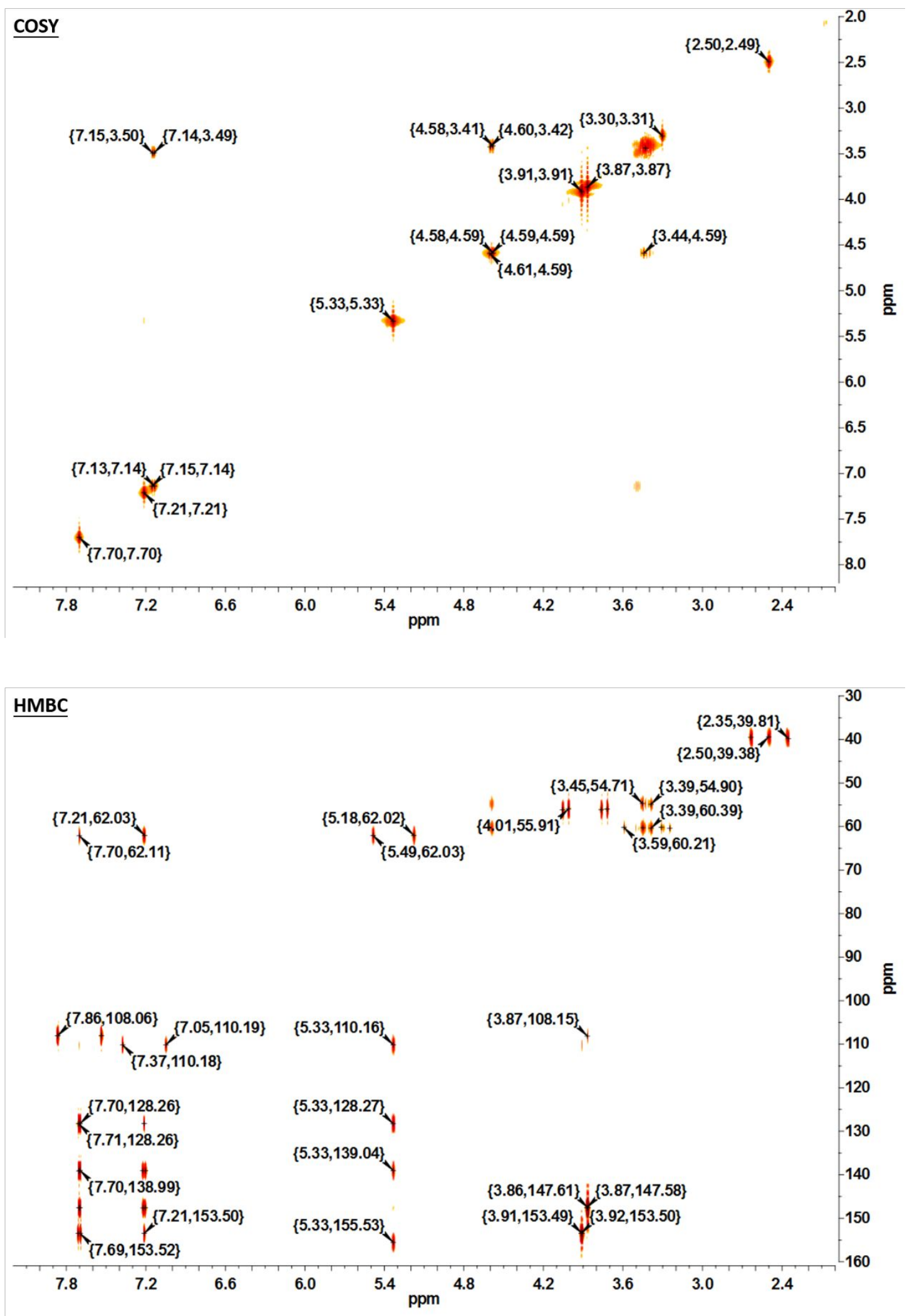

Figure S3. COSY and HMBC NMR spectra of compound 3 


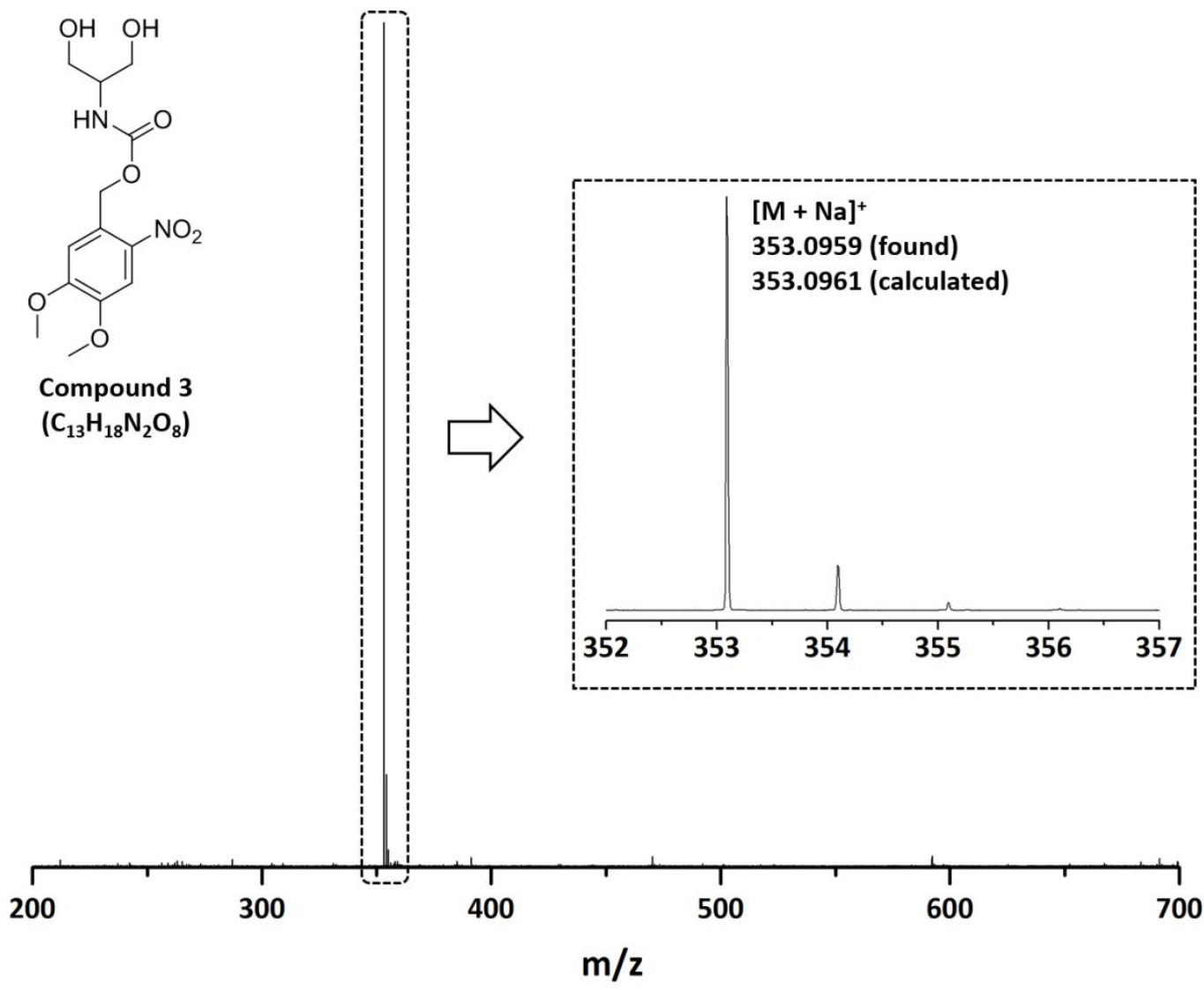

Figure S4. ESI-ToF-mass spectrum of compound 3

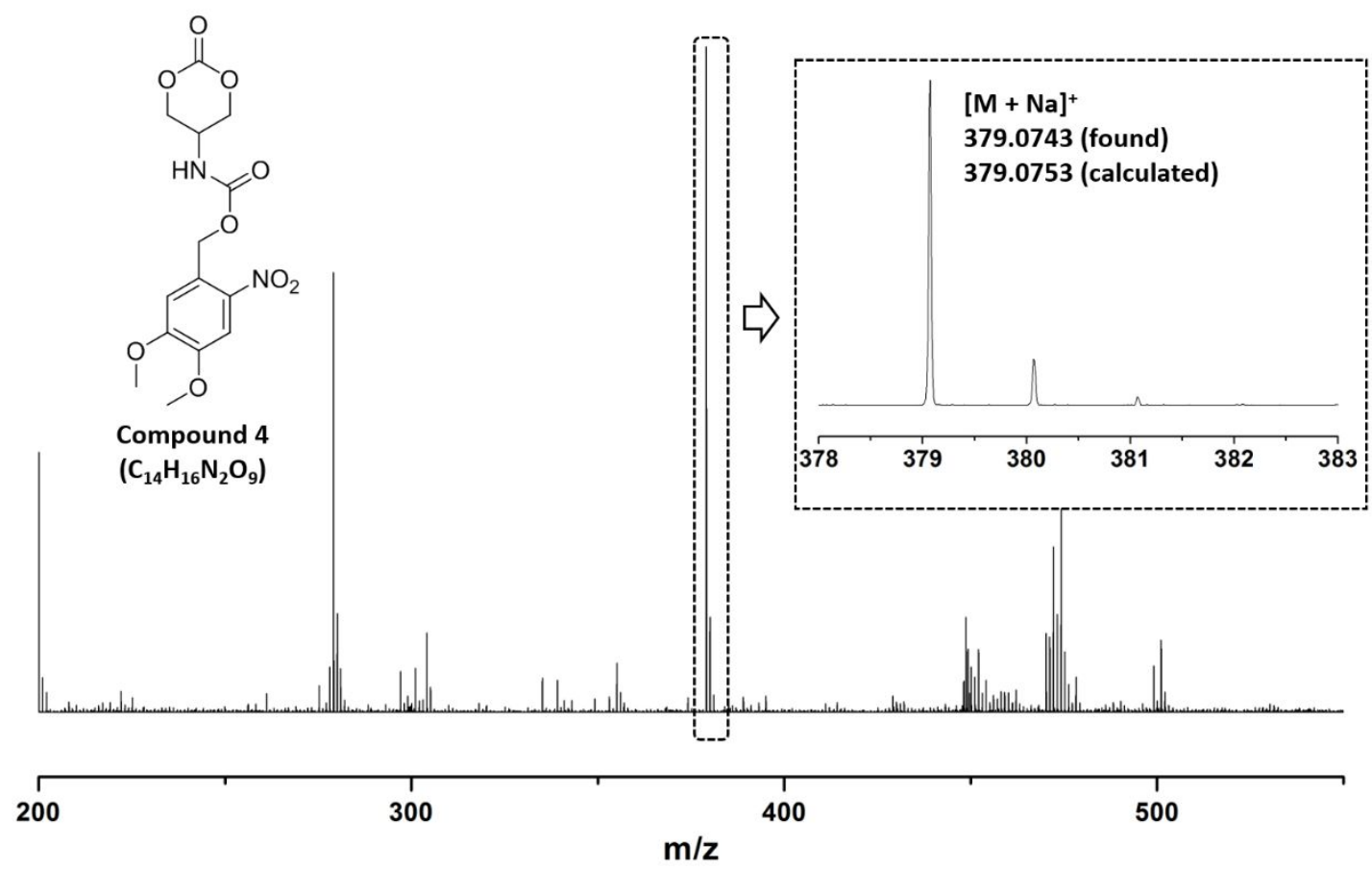

Figure S5. ESI-ToF-mass spectrum of compound 4 

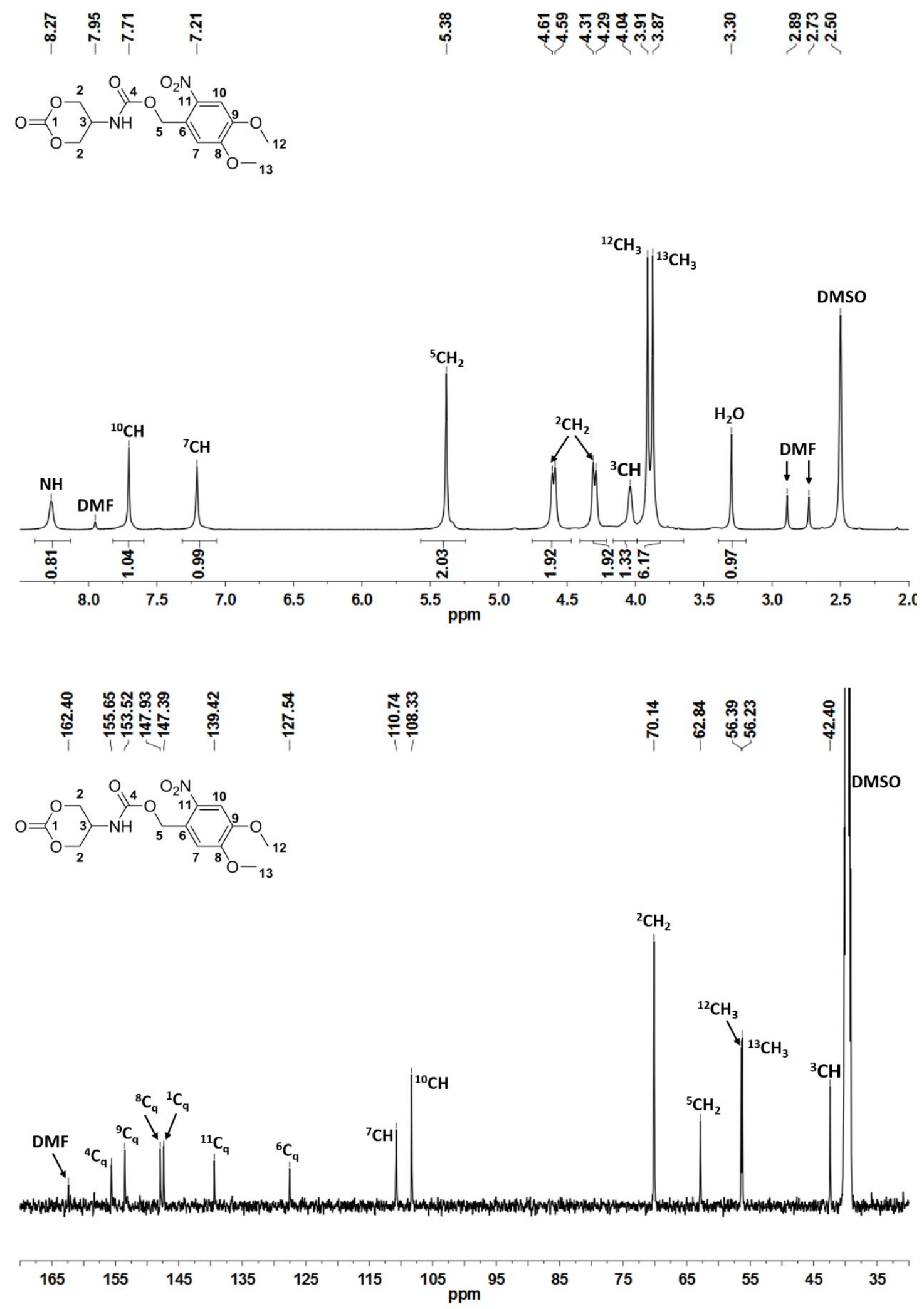

Figure S6. ${ }^{1} \mathrm{H}$ and ${ }^{13} \mathrm{C}$ NMR spectra of compound 4 
a)<smiles>COc1cc(COC(=O)NC2COC(=O)OC2)c([N+](=O)[O-])cc1OC</smiles><smiles>COc1cc(COC(=O)NC2COC(=O)OC2)c([N+](=O)[O-])cc1OC</smiles><smiles>COc1cc(COC(=O)N(C2COC(=O)OC2)C2COC(=O)O2)c([N+](=O)[O-])cc1OC</smiles>

b)<smiles>COc1cc(COC(=O)NC2COC(=O)OC2)c([N+](=O)[O-])cc1OC</smiles><smiles>COc1cc(COC(=O)NC2CCCCCC(=O)OC2)c([N+](=O)[O-])cc1OC</smiles>
cyclization<smiles>COc1cc(COC(=O)N2CC(CO)OC2=O)c([N+](=O)[O-])cc1OC</smiles>

c)<smiles>COc1cc(COC(=O)NC(COC(C)=O)COC(C)=O)c([N+](=O)[O-])cc1OC</smiles><smiles>CCC(=O)OCC(COC)N1CCCCC1COC(=O)OCc1cc(OC)c(OC)cc1[N+](=O)[O-]</smiles>

intramolecular cyclization

degradation products

Scheme S6. Possible mechanisms of the side reaction during the ROP of monomer 4:

a) formation of oligomers initiated by deprotonated carbamate; b) formation of fivemembered transcarbamation product from monomer $\mathbf{4}$ and c) DBU-catalyzed degradation of oligomer by intramolecular transcarbamation. 


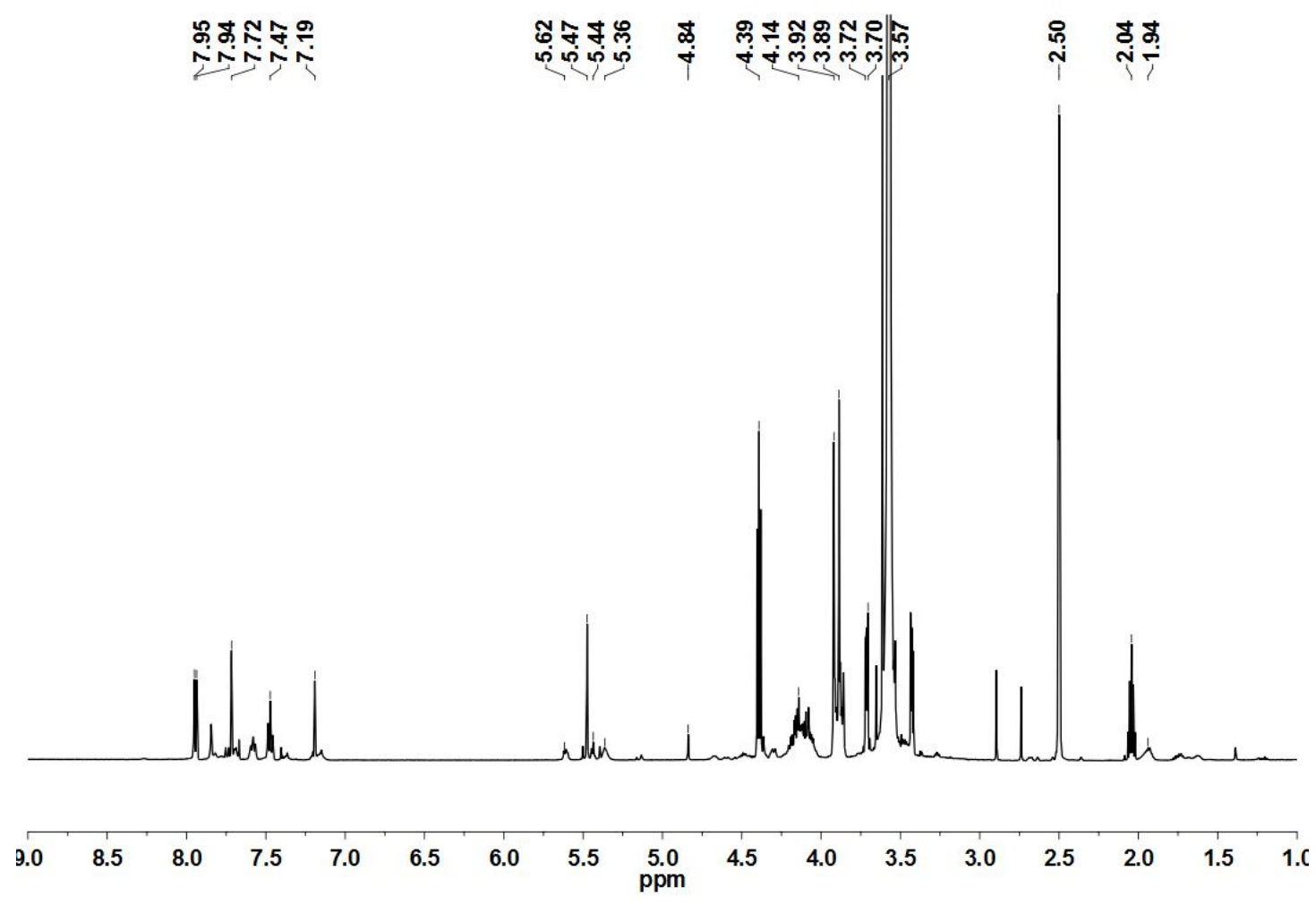

Figure S7. ${ }^{1} \mathrm{H}$ NMR spectrum of ROP solution after $3.5 \mathrm{~h}$

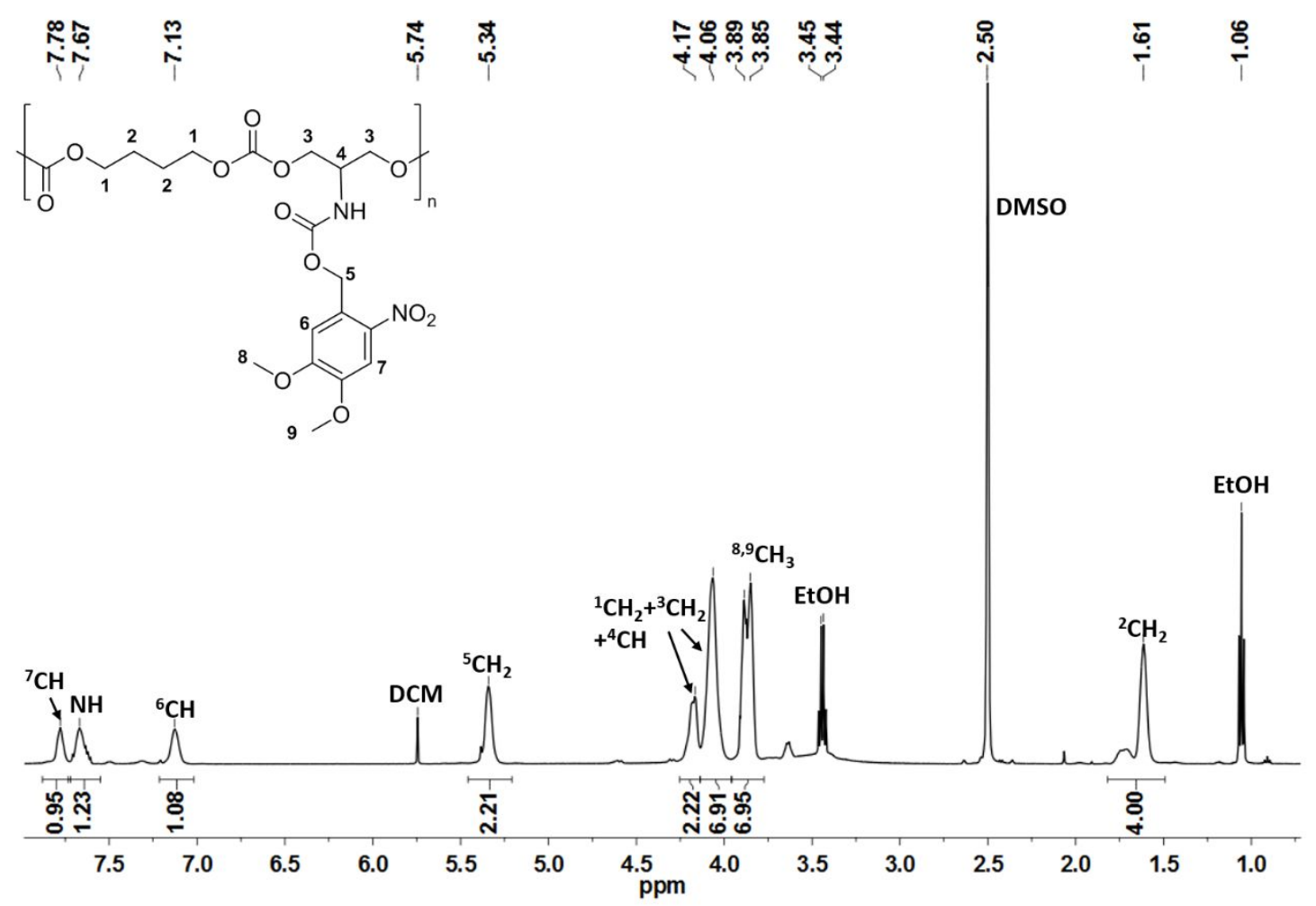

Figure S8. ${ }^{1} \mathrm{H}$ NMR spectrum of LrPC 


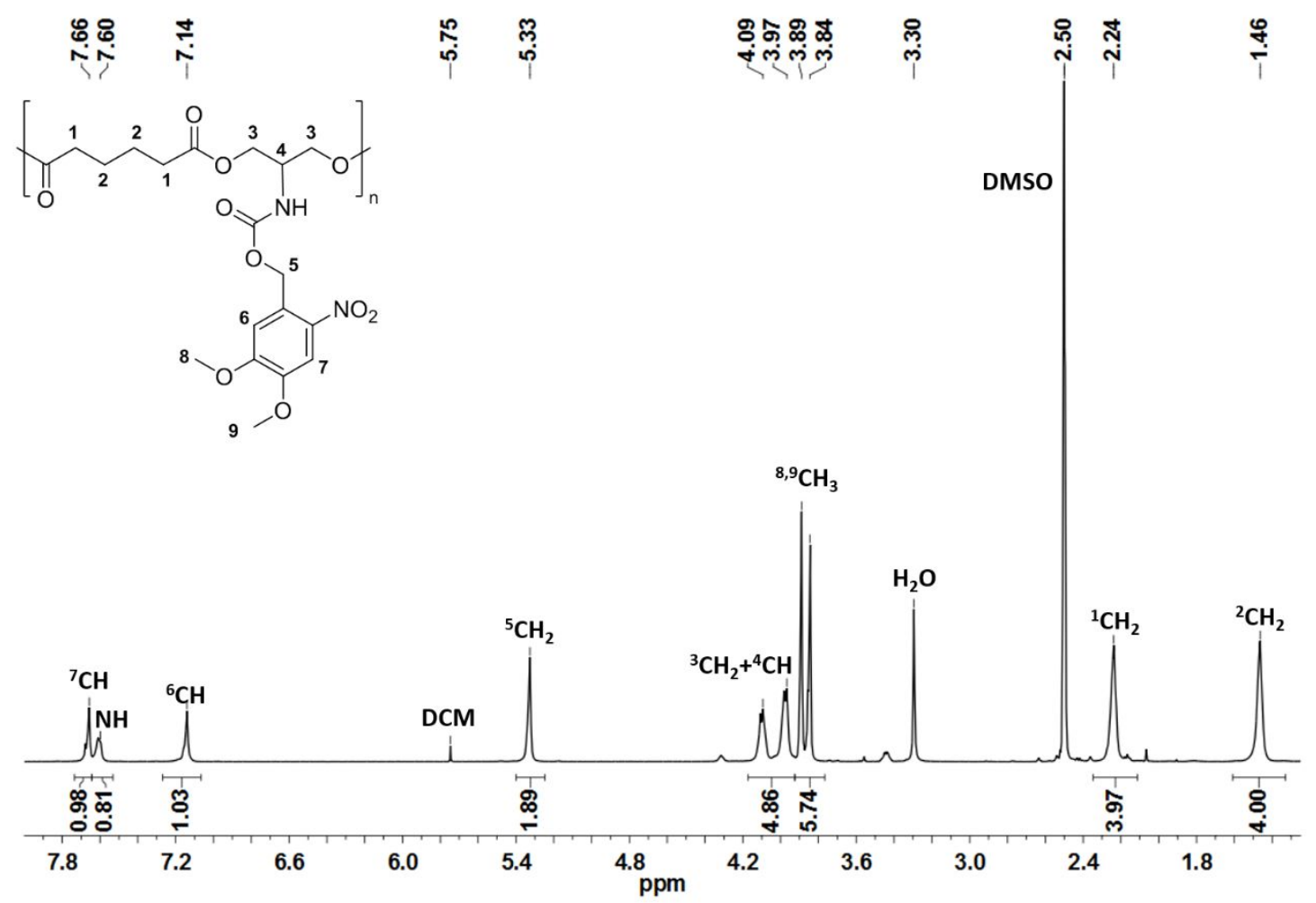

Figure S9. ${ }^{1} \mathrm{H}$ NMR spectrum of LrPE

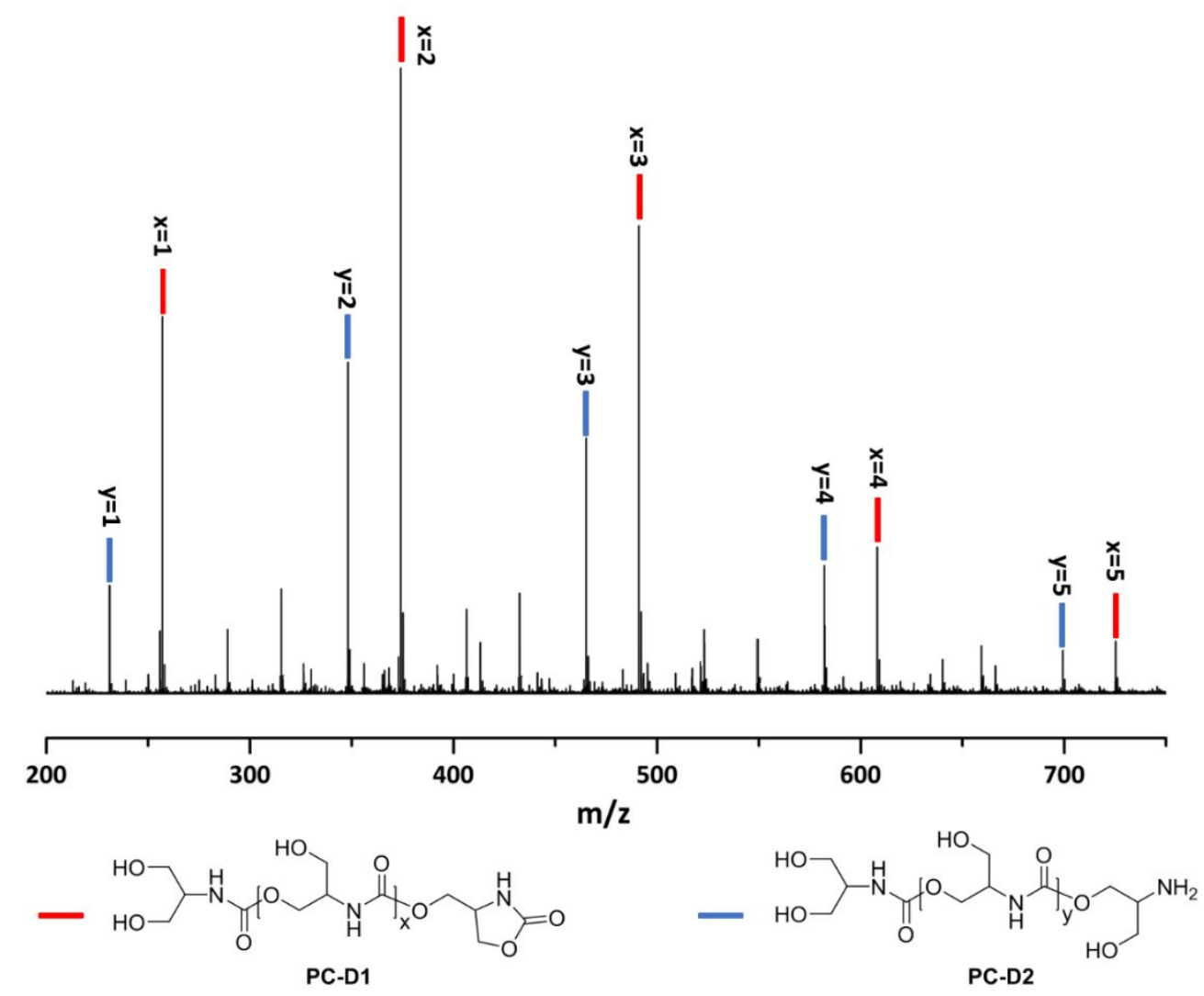

Figure S10. ESI-ToF mass spectrum of degradation products from PC-Boc after deprotection with $\mathrm{HCl} / 1$,4-dioxane solution and neutralization with TEA. 
Table S1. Mass spectrometry results of PC-D1 and PC-D2

\begin{tabular}{|c|c|c|c|c|}
\hline & & $\begin{array}{c}\text { Calculated } \\
{[\mathrm{m} / \mathrm{z}]} \\
{[\mathrm{M}+\mathrm{Na}]^{+}} \\
\end{array}$ & $\begin{array}{c}\text { Found } \\
{[\mathrm{m} / \mathrm{z}]} \\
{[\mathrm{M}+\mathrm{Na}]^{+}}\end{array}$ & $\begin{array}{c}\text { Accuracy } \\
{[\mathrm{ppm}]}\end{array}$ \\
\hline $\mathrm{HO}-$ & $\mathrm{x}=1$ & 257.0744 & 257.0822 & 30 \\
\hline $\mathrm{HO}-$ & $x=2$ & 374.1170 & 374.1243 & 19 \\
\hline PC-D1 & $x=3$ & 491.1596 & 491.1666 & 14 \\
\hline & $x=4$ & 608.2022 & 608.2062 & 7 \\
\hline & $x=5$ & 725.2448 & 725.2449 & 0.1 \\
\hline & $y=1$ & 231.0951 & 231.0978 & 12 \\
\hline 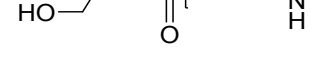 & $y=2$ & 348.1377 & 348.1435 & 17 \\
\hline \multirow[t]{3}{*}{ PC-D2 } & $y=3$ & 465.1803 & 465.1876 & 16 \\
\hline & $y=4$ & 582.2229 & 582.2281 & 9 \\
\hline & $y=5$ & 699.2655 & 699.2765 & 16 \\
\hline
\end{tabular}
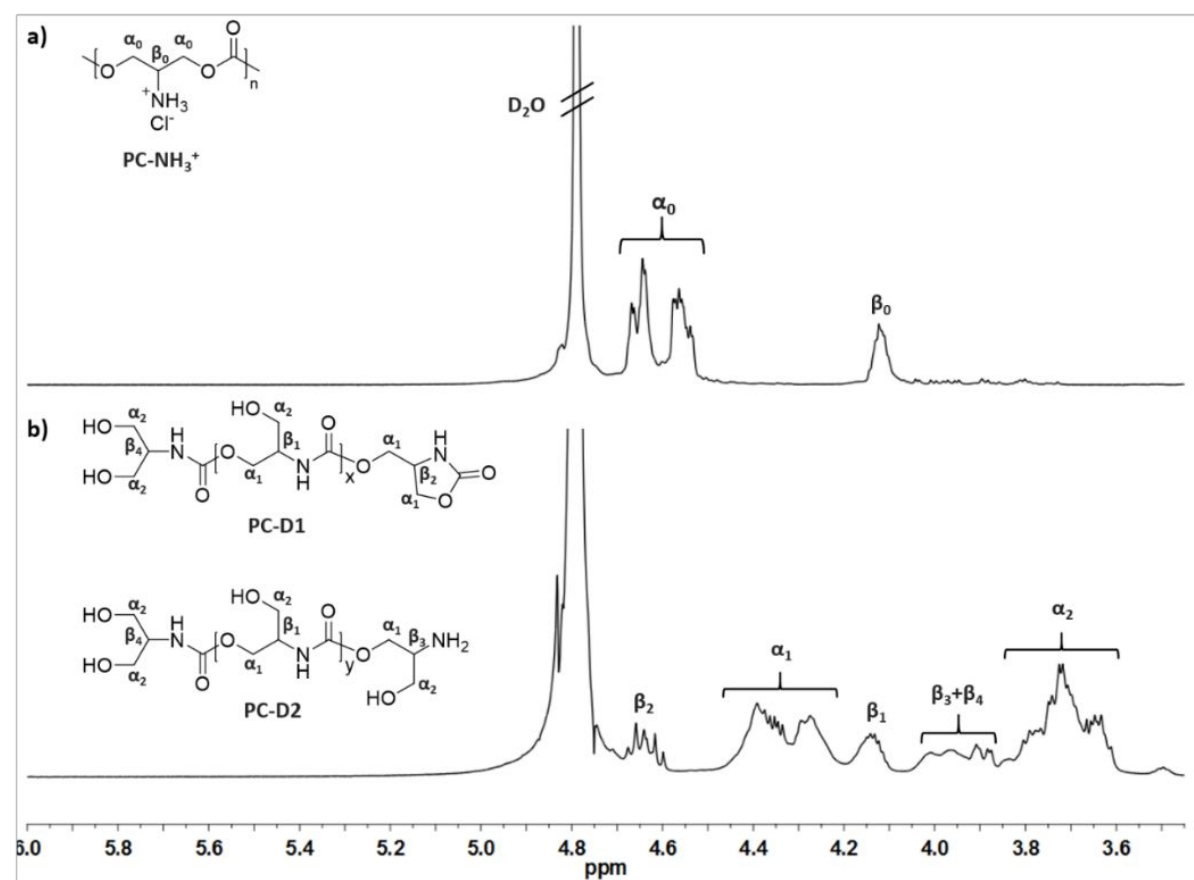

Figure S11. ${ }^{1} \mathrm{H}$ NMR spectra of $\mathrm{PC}-\mathrm{NH}_{3}{ }^{+}$(a) and degradation products $\mathrm{PC}-\mathrm{D} 1$ and PCD2 (b). 


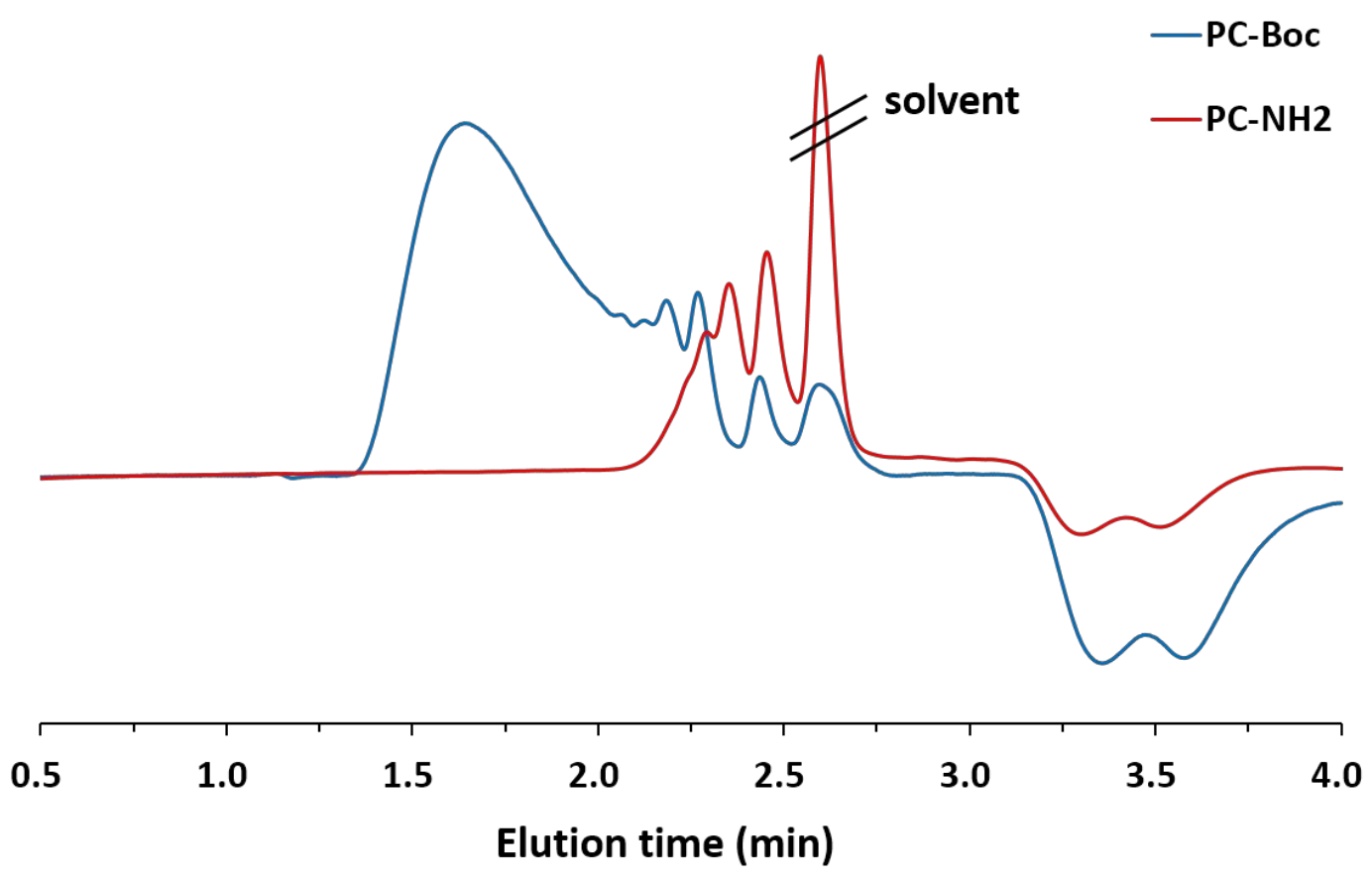

Figure S12. SEC traces of PC-Boc and $\mathrm{PC}-\mathrm{NH}_{2}$.

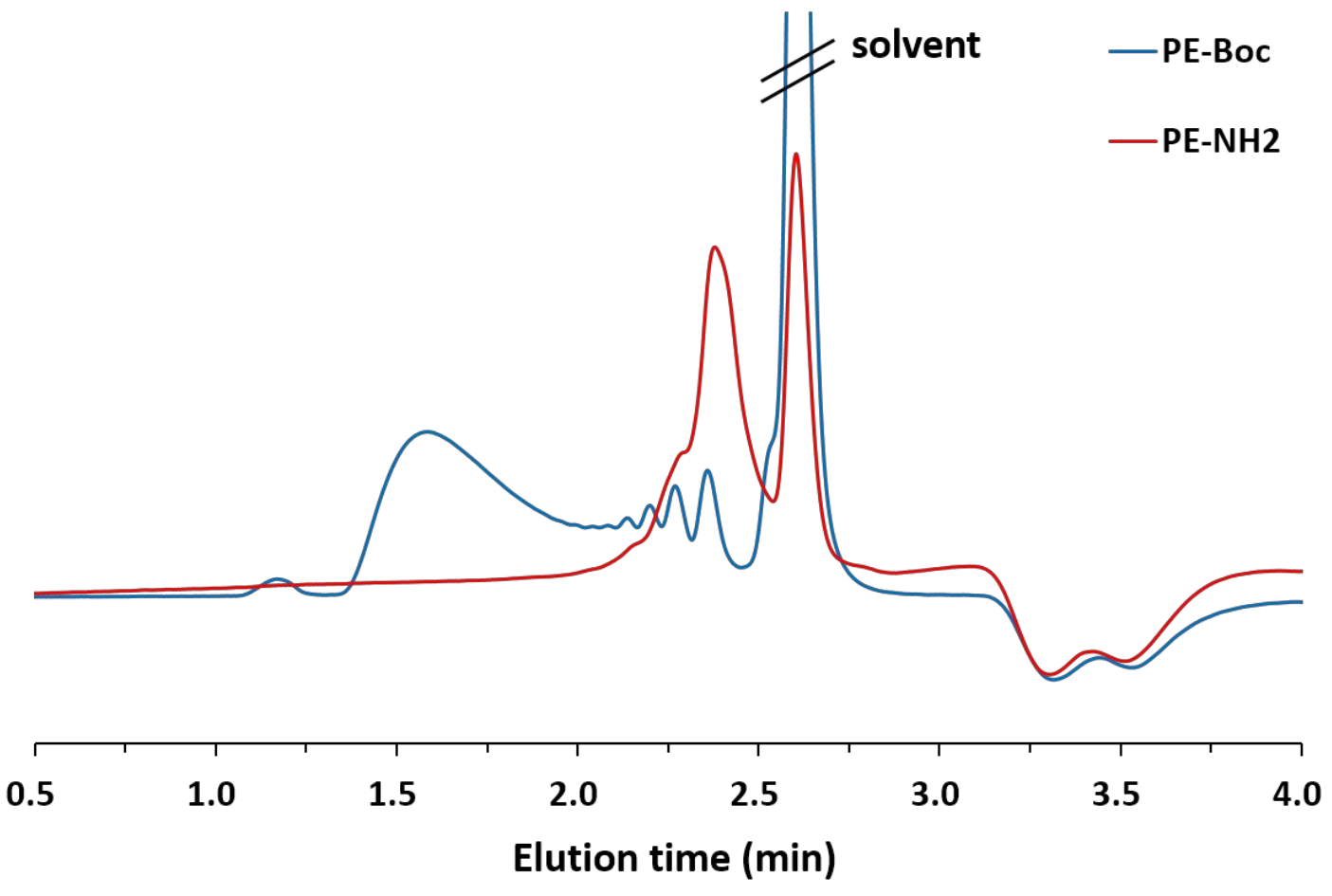

Figure S13. SEC traces of PE-Boc and $\mathrm{PE}-\mathrm{NH}_{2}$. 


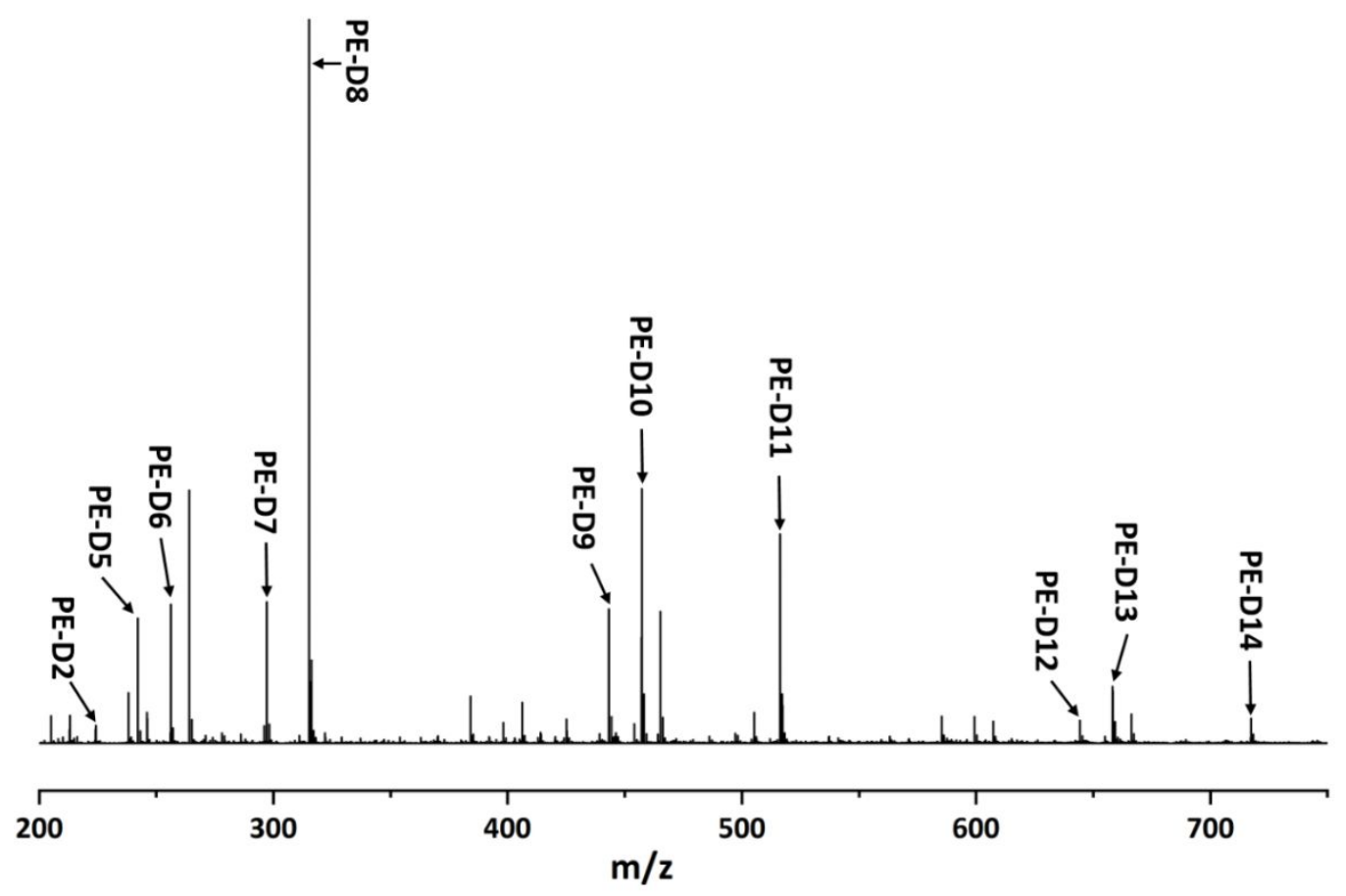

Figure S14. ESI-ToF mass spectrum of degradation products from PE-Boc after deprotection with $\mathrm{HCl} / 1$,4-dioxane solution and neutralization with TEA.

Table S2. Mass spectrometry results of degradation products for $\mathrm{PE}-\mathrm{NH}_{2}$

\begin{tabular}{|c|c|c|c|}
\hline Degradation products & $\begin{array}{c}\text { Calculated } \\
{[\mathrm{m} / \mathrm{z}]} \\
{[\mathrm{M}+\mathrm{Na}]^{+}}\end{array}$ & $\begin{array}{c}\text { Found } \\
{[\mathrm{m} / \mathrm{z}]} \\
{[\mathrm{M}+\mathrm{Na}]^{+}}\end{array}$ & $\begin{array}{c}\text { Accuracy } \\
{[\mathrm{ppm}]}\end{array}$ \\
\hline $\begin{array}{l}\text { PE-D1 } \\
\end{array}$ & 114.0525 & $\begin{array}{c}\text { Not } \\
\text { found }\end{array}$ & - \\
\hline PE-D2 & 224.0893 & 224.0934 & 18 \\
\hline PE-D3 & 352.1367 & $\begin{array}{l}\text { Not } \\
\text { found }\end{array}$ & - \\
\hline PE-D4 & 366.1523 & $\begin{array}{l}\text { Not } \\
\text { found }\end{array}$ & - \\
\hline
\end{tabular}




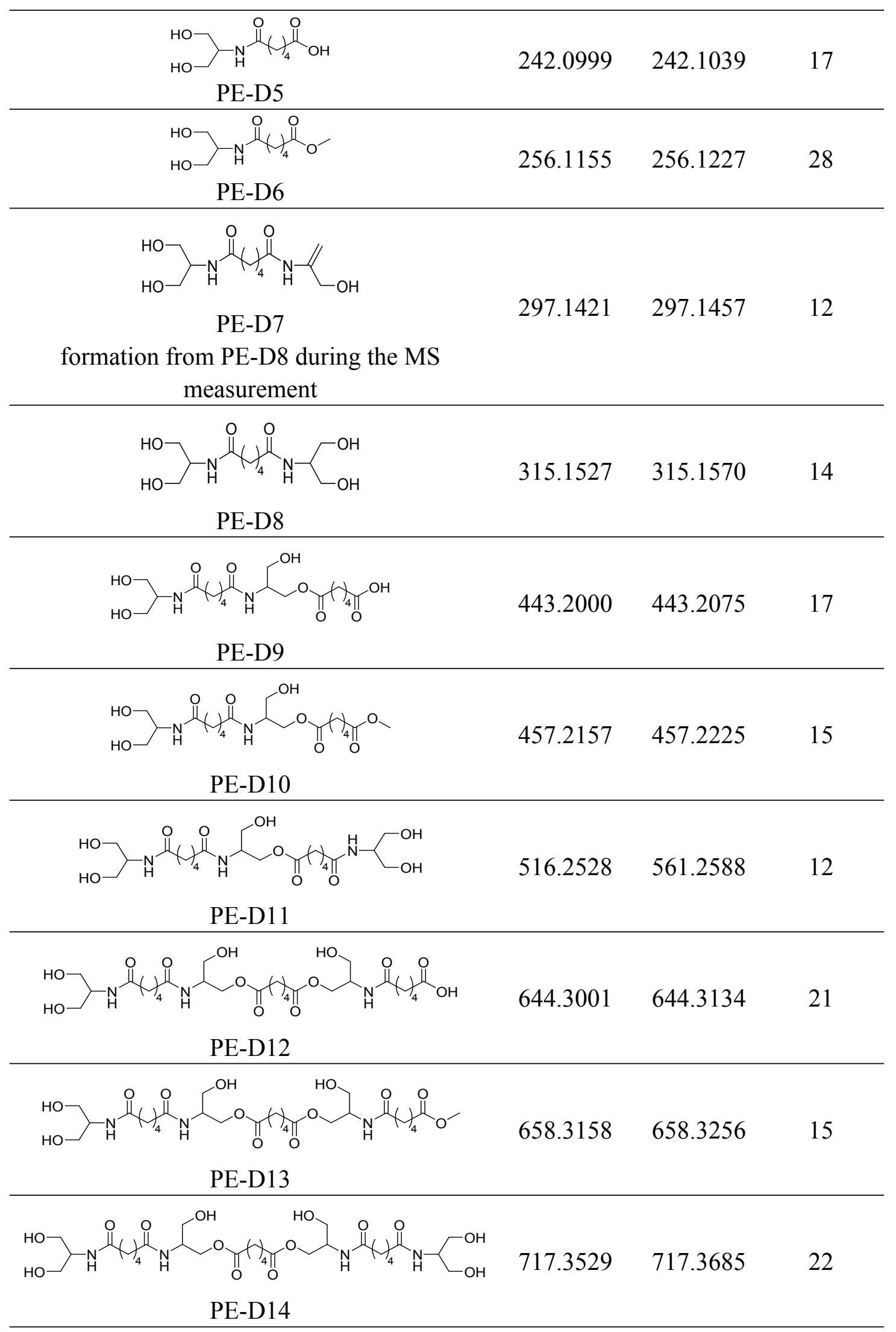




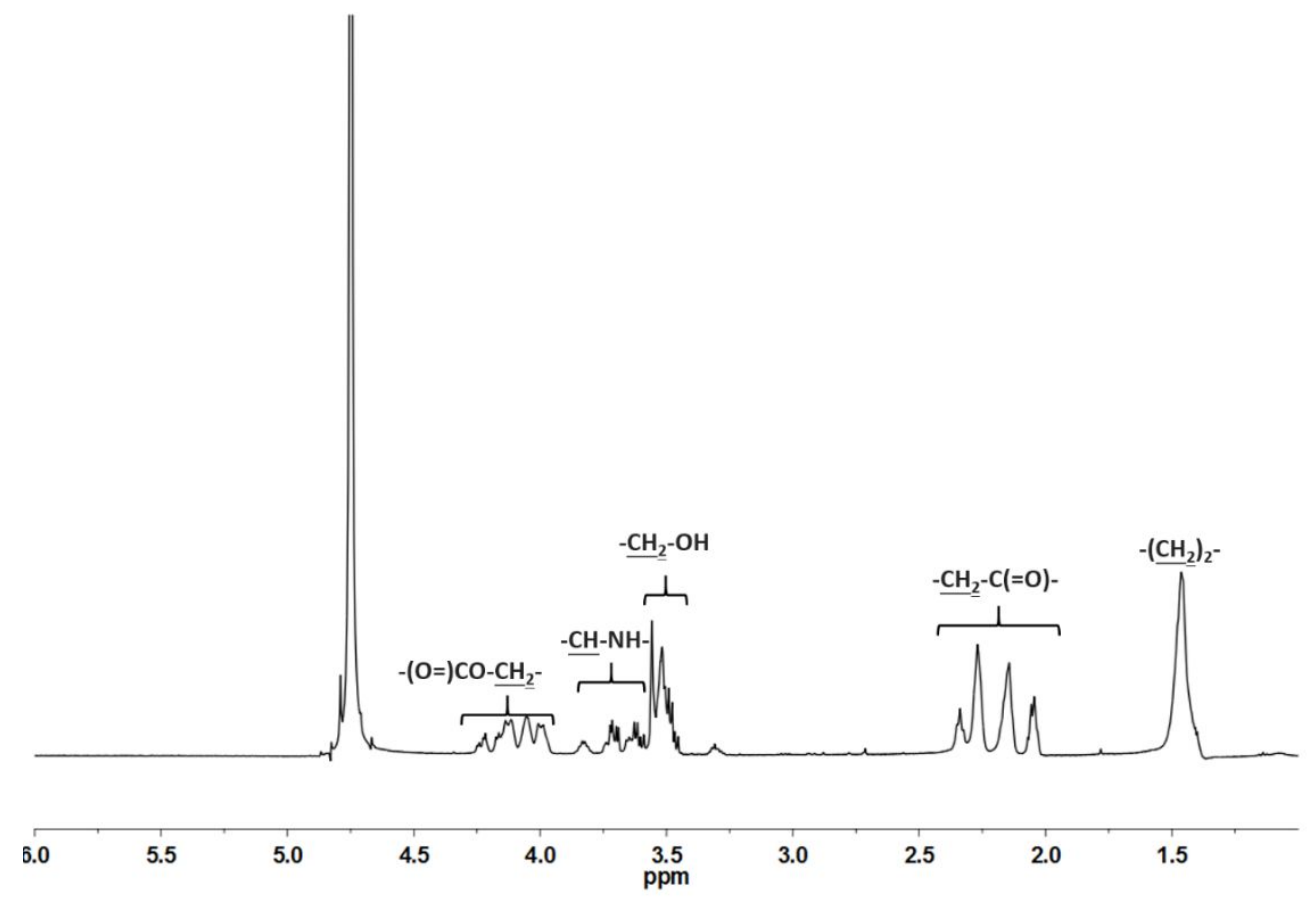

Figure S15. ${ }^{1} \mathrm{H}$ NMR spectrum of degradation products of PE-Boc after deprotection and neutralization.

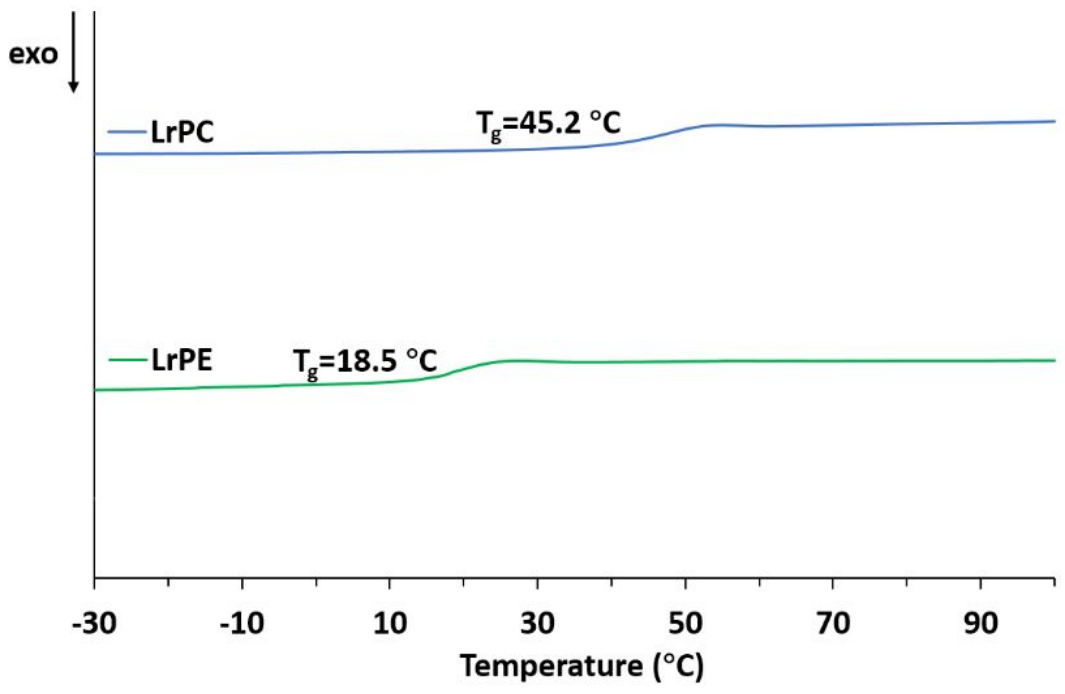

Figure S16. DSC traces of LrPC and LrPE from second heating cycle at $10 \mathrm{~K} / \mathrm{min}$. 
- Additional ESI-ToF mass spectrum and NMR spectra of small molecules and polymers
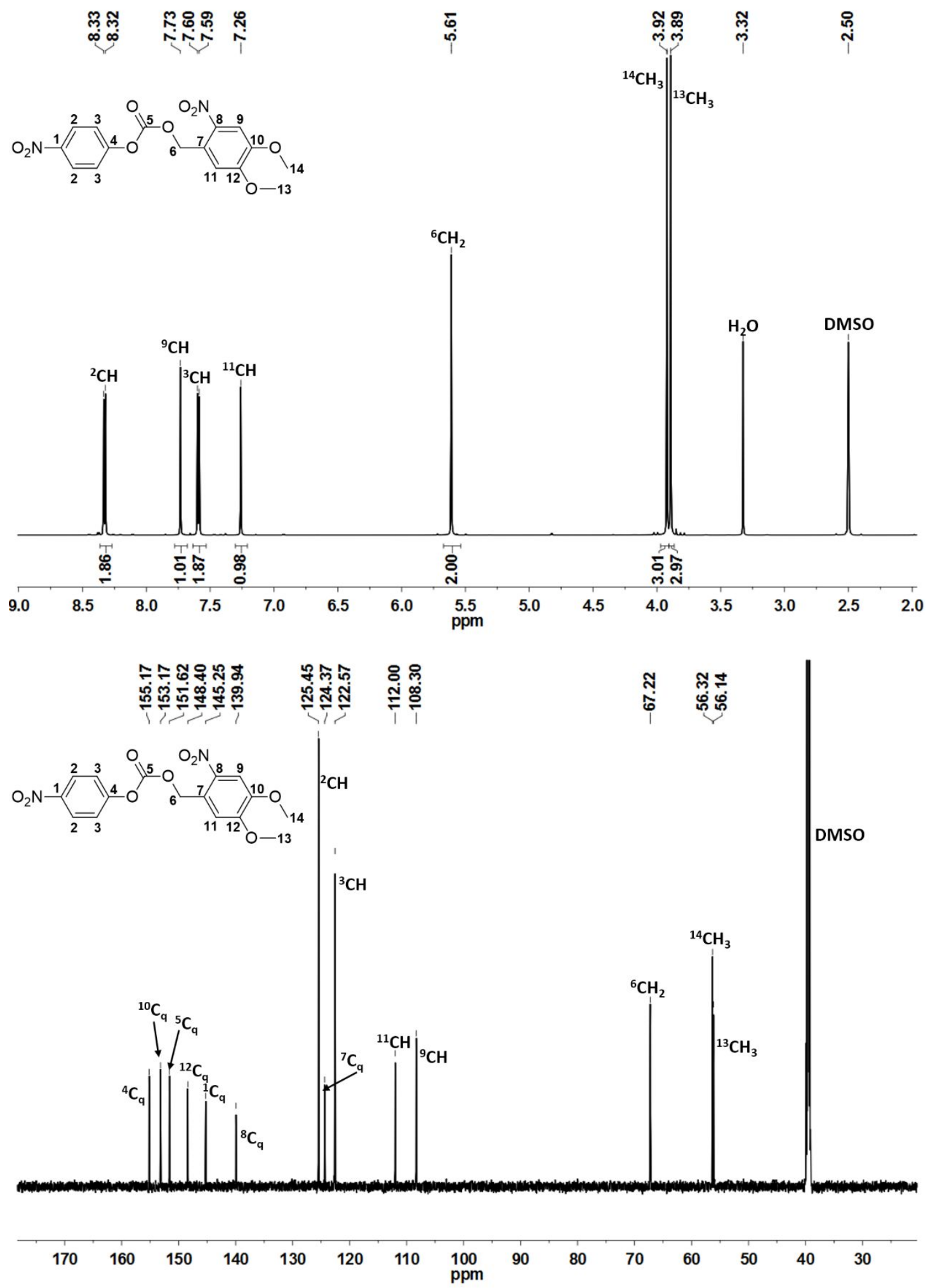

Figure S17. ${ }^{1} \mathrm{H}$ and ${ }^{13} \mathrm{C}$ NMR spectra of compound 2 

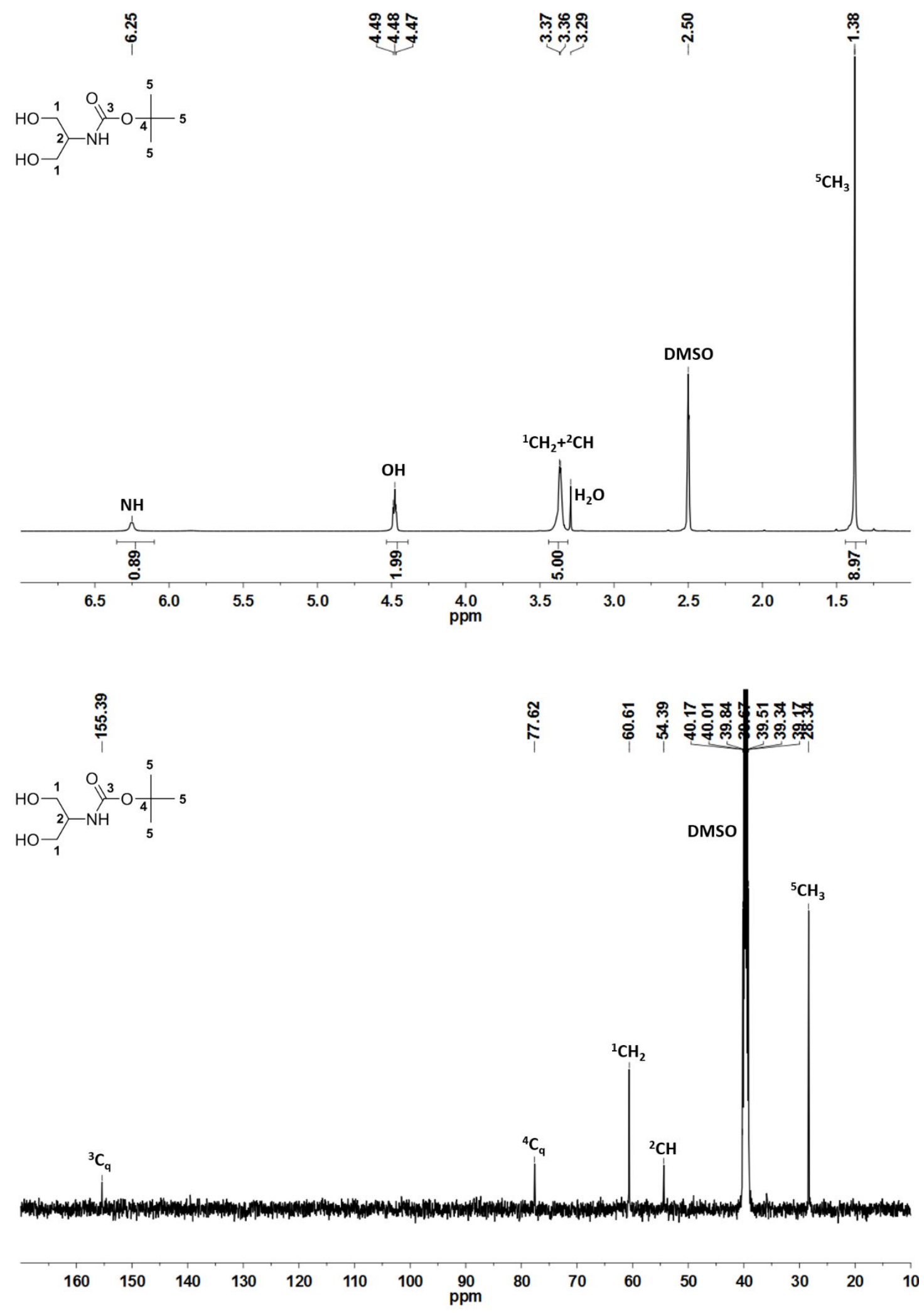

Figure S18. ${ }^{1} \mathrm{H}$ and ${ }^{13} \mathrm{C}$ NMR spectra of compound 5 


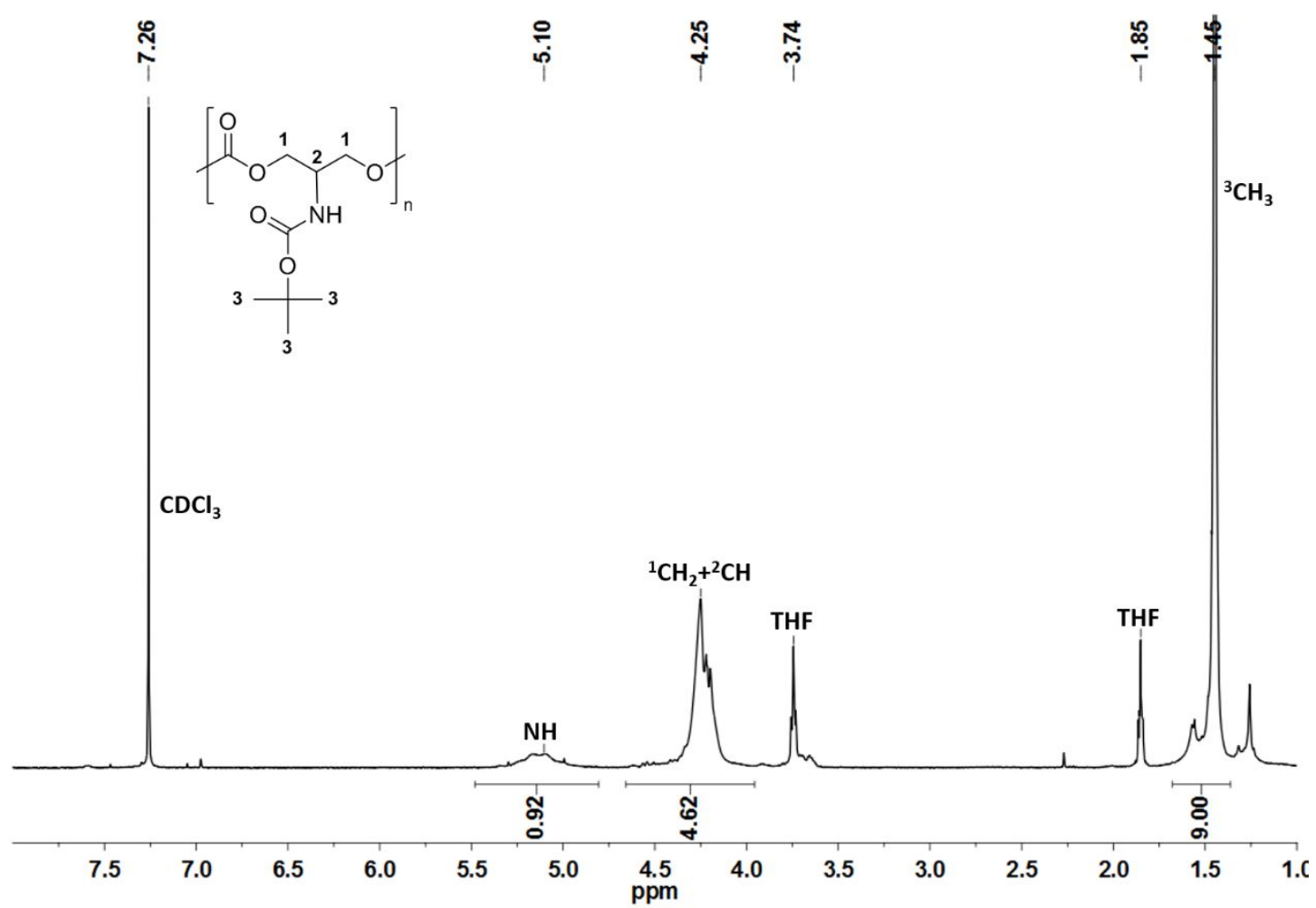

Figure S19. ${ }^{1} \mathrm{H}$ NMR spectrum of PC-Boc
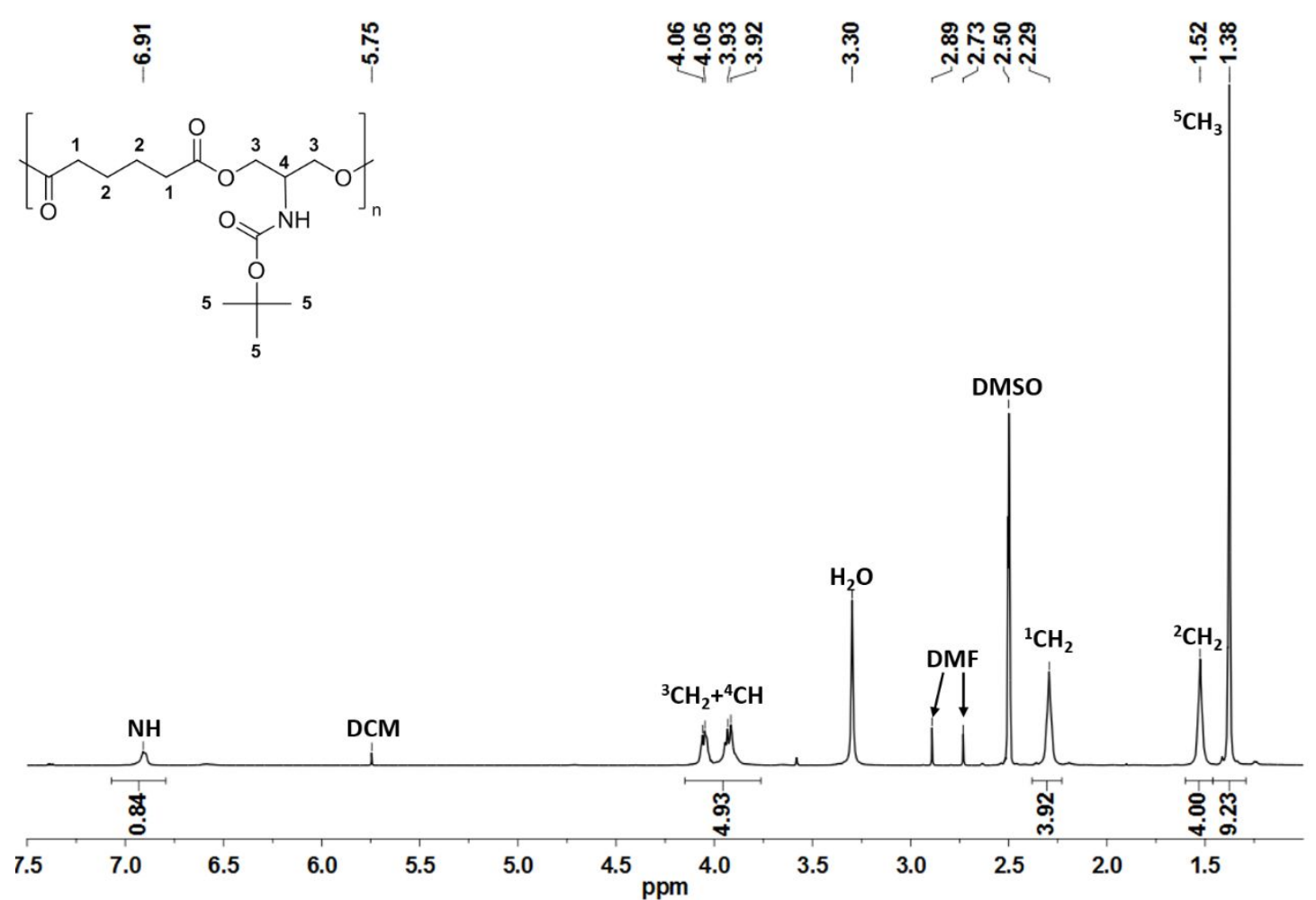

Figure S20. ${ }^{1} \mathrm{H}$ NMR spectrum of PE-Boc 


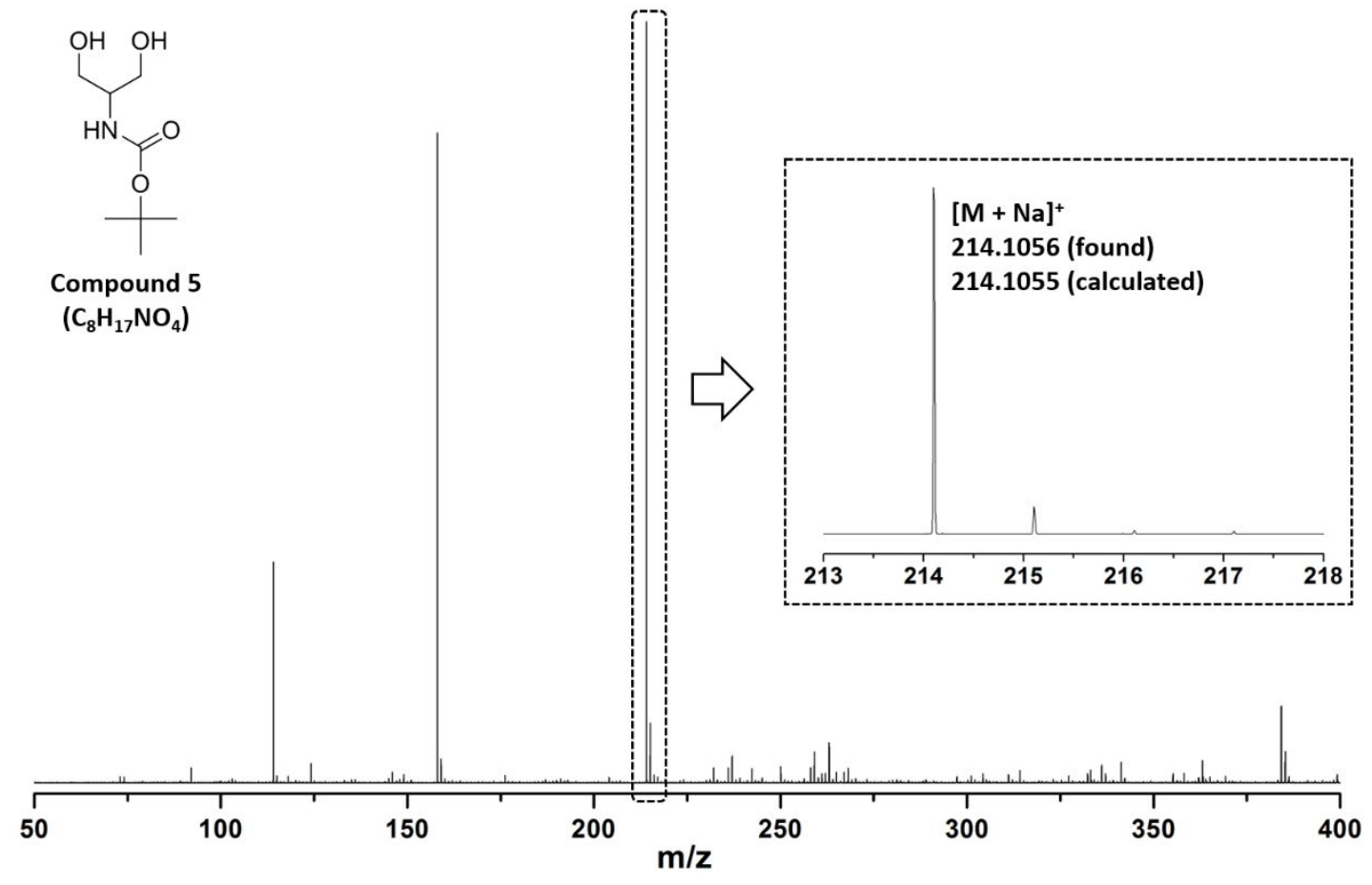

Figure S21. ESI-ToF-mass spectrum of compound 5

\section{- Reference}

1. Fomina, N.; McFearin, C.; Sermsakdi, M.; Edigin, O.; Almutairi, A. UV and NearIR Triggered Release from Polymeric Nanoparticles. J. Am. Chem. Soc. 2010, 132 (28), 9540-9542.

2. Qiu, F.; Zhang, M.; Du, F.; Li, Z. Oxidation Degradable Aliphatic Polycarbonates with Pendent Phenylboronic Ester. Macromolecules 2017, 50 (1), 23-34. 